\title{
Drawing Outer 1-planar Graphs with Few Slopes
}

\author{
Emilio Di Giacomo Giuseppe Liotta Fabrizio Montecchiani
}

Dip. di Ingegneria, Università degli Studi di Perugia

\begin{abstract}
A graph is outer 1-planar if it admits a drawing where each vertex is on the outer face and each edge is crossed by at most another edge. Outer 1-planar graphs are a superclass of the outerplanar graphs and a subclass of the planar partial 3-trees. We show that an outer 1-planar graph $G$ of bounded degree $\Delta$ admits an outer 1-planar straight-line drawing that uses $O(\Delta)$ different slopes, which generalizes a previous result by Knauer et al. about the outerplanar slope number of outerplanar graphs [18. We also show that $O\left(\Delta^{2}\right)$ slopes suffice to construct a crossing-free straight-line drawing of $G$; the best known upper bound on the planar slope number of planar partial 3-trees of bounded degree $\Delta$ is $O\left(\Delta^{5}\right)$ as proved by Jelínek et al. [16.
\end{abstract}

\begin{tabular}{|ccccc|}
\hline Submitted: & Reviewed: & Revised: & Reviewed: & Revised: \\
October 2014 & February 2015 & March 2015 & August 2015 & September 2015 \\
& Accepted: & Final: & Published: \\
& October 2015 & October 2015 & November 2015 \\
& Article type: & & Communicated by: \\
& Regular paper & & C. Duncan and A. Symvonis \\
\hline
\end{tabular}

Research supported in part by the MIUR project AMANDA "Algorithmics for MAssive and Networked DAta", prot. 2012C4E3KT_001.

E-mail addresses: emilio.digiacomo@unipg.it (Emilio Di Giacomo) giuseppe.liotta@unipg.it (Giuseppe Liotta) fabrizio.montecchiani@unipg.it (Fabrizio Montecchiani) 


\section{Introduction}

The slope number of a graph $G$ is defined as the minimum number of distinct edge slopes required to construct a straight-line drawing of $G$. Minimizing the number of slopes used in a straight-line graph drawing is a desirable aesthetic requirement and an interesting theoretical problem which has received considerable attention since its first definition by Wade and Chu [26]. Let $\Delta$ be the maximum degree of a graph $G$ and let $m$ be the number of edges of $G$, then the slope number of $G$ is at least $\left\lceil\frac{\Delta}{2}\right\rceil$ and at most $m$.

For non-planar graphs, there exist graphs with $\Delta \geq 5$ whose slope number is unbounded with respect to $\Delta[4,22$, while the slope number of graphs with $\Delta=4$ is unknown, and the slope number of graphs with $\Delta=3$ is four 21 .

Concerning planar graphs, the planar slope number of a planar graph $G$ is defined as the minimum number of distinct slopes required by any planar straight-line drawing of $G$ (see, e.g., [1]). Keszegh, Pach, and Pálvölgyi [17] prove that $O\left(2^{O(\Delta)}\right)$ is an upper bound and that $3 \Delta-6$ is a lower bound for the planar graphs of bounded degree $\Delta$. The gap between upper and lower bound has been reduced for special families of planar graphs with bounded degree. Knauer, Micek, and Walczak [18, prove that an outerplanar graph of bounded degree $\Delta \geq 4$ admits an outerplanar straight-line drawing that uses at most $\Delta-1$ distinct edge slopes, and this bound is tight. Moreover, the slope number of planar partial 3-trees of bounded degree $\Delta$ is $O\left(\Delta^{5}\right)$, as shown by Jelínek et al. [16, while all partial 2-trees of bounded degree $\Delta$ have $O(\Delta)$ slope number, which is a result by Lenhart et al. 20. Di Giacomo et al. [9] show that planar graphs with at least five vertices and bounded degree $\Delta \leq 3$ have planar slope number four, which is worst case optimal.

The research in this paper is motivated by the following observations. The fact that the best known upper bound on the planar slope number is $O\left(\Delta^{5}\right)$ for planar partial 3-trees, while it is $O(\Delta)$ for partial 2-trees, suggests to further investigate the planar slope number of those planar graphs whose treewidth is

at most three. Also, the fact that non-planar drawings may require a number of slopes that is unbounded in $\Delta$ while the planar slope number of planar graphs is bounded in $\Delta$, suggests to study how many slopes may be needed to construct straight-line drawings that are "nearly-planar" in some sense, i.e., where only some types of edge crossings are allowed.

We study outer 1-planar graphs, which are graphs that admit drawings where each edge is crossed at most once and each vertex is on the boundary of the outer face. This family of graphs has recently received remarkable attention in the general research framework of "graph drawing beyond planarity" (see, e.g., 2, 6, 14]). In particular, in 2013, Auer et al. 2, and independently Hong et al. 14, presented a linear-time algorithm to test outer 1-planarity. Both algorithms produce an outer 1-planar embedding of the graph if it exists. Also, outer 1-planar graphs are known to be planar graphs and they have treewidth at most three [2.

Given an outer 1-planar graph $G$, we define the outer 1-planar slope number of $G$ as the minimum number of distinct slopes required by any outer 1-planar 


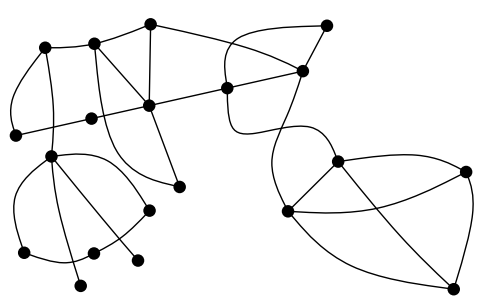

(a)

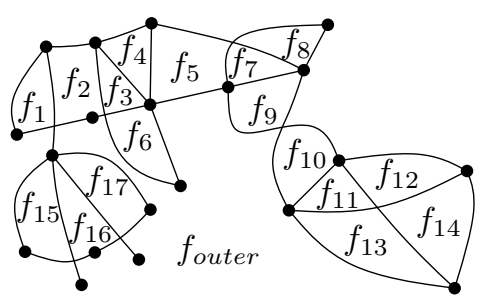

(b)

Figure 1: (a) An outer 1-planar drawing $\Gamma$ of an outer 1-planar graph $G$. (b) Illustration of the faces defined in the embedding $\mathcal{E}(G)$ of $G$.

straight-line drawing of $G$. We prove the following results.

1. We study planar straight-line drawings of outer 1-planar graphs of bounded degree $\Delta$ and show an $O\left(\Delta^{2}\right)$ upper bound for the planar slope number. Hence, for this special family of planar partial 3-trees, we are able to reduce the general $O\left(\Delta^{5}\right)$ upper bound [16].

2. We show that the outer 1-planar slope number of outer 1-planar graphs with maximum degree $\Delta$ is at most $6 \Delta+12$. This result goes in the direction of studying how many slopes may be needed to construct straight-line drawings that are "nearly-planar". Moreover, since outerplanar drawings are a special case of the outer 1-planar drawings, this result generalizes the above mentioned upper bound on the (outer)planar slope number of outerplanar graphs [18.

Our results are constructive and give rise to linear-time drawing algorithms in the real RAM model of computation. Also, it may be worth recalling that the study of 1-planar graphs, i.e., those graphs that can be drawn with at most one crossing per edge, has recently received a lot of interest (see, e.g., 1, 3, 5], 6, 10, 12, 13, 15, 19, 23, 25]).

In Section 2 we give preliminary definitions and basic properties of outer 1planar graphs. The planar slope number of outer 1-planar graphs is investigated in Section 3, while their outer 1-planar slope number is studied in Section 4. Section 5 lists some open problems.

\section{Preliminaries and Basic Definitions}

Basic Definitions. A graph $G$ is simple if contains neither loops nor multiple edges. Also, $G$ is undirected if its edges are not oriented. In this paper we only consider simple, undirected graphs. A drawing $\Gamma$ of a graph $G=(V, E)$ is a mapping of the vertices in $V$ to points of the plane, and of the edges in $E$ to Jordan arcs connecting their corresponding endpoints but not passing through any other vertex. We only consider simple drawings, i.e., drawings such that 
two arcs representing two edges have at most one point in common, and this point is either a common endpoint or a common interior point where the two arcs properly cross each other. $\Gamma$ is a straight-line drawing if every edge is mapped to a straight-line segment. $\Gamma$ is a planar drawing if no edge is crossed; it is a 1-planar drawing if each edge is crossed at most once. A planar graph is a graph that admits a planar drawing; a 1-planar graph is a graph that admits a 1-planar drawing.

A planar drawing of a graph subdivides the plane into topologically connected regions, called faces. The unbounded region is called the outer face. A planar embedding $\mathcal{E}(G)$ of a planar graph $G$ is an equivalence class of planar drawings that define the same set of faces. A planar embedding is described by the circular list of the edges around each vertex together with the choice of the outer face. The concept of planar embeddings can be extended to 1-planar drawings as follows. Given a 1-planar drawing $\Gamma$ we can planarize it by replacing each crossing with a dummy vertex. Let $\Gamma^{*}$ be the resulting planarized drawing, then the (curves representing the) edges of $\Gamma^{*}$ are called edge fragments of $G$. Note that an edge fragment corresponds either to a portion of a real edge connecting a vertex to a crossing, or to a real edge connecting two vertices. In the latter case the fragment is said to be trivial. The planarized drawing $\Gamma^{*}$ subdivides the plane into topologically connected regions, called faces. A 1planar embedding $\mathcal{E}(G)$ of a 1-planar graph $G$ is an equivalence class of 1-planar drawings whose planarized versions define the same set of faces. An outerplanar drawing is a planar drawing with all vertices on the outer face. An outerplanar graph is a graph admitting an outerplanar drawing. An outer 1-planar drawing is a 1-planar drawing with all vertices on the outer face. An outer 1-planar graph is a graph admitting an outer 1-planar drawing. An outer 1-plane graph $G$ is an outer 1-planar graph with a given outer 1-planar embedding $\mathcal{E}(G)$. See also Figure 1 for an example.

The slope $s$ of a line $\ell$ is the angle that a horizontal line needs to be rotated counter-clockwise in order to make it overlap with $\ell$. The slope of a segment representing an edge in a straight-line drawing is the slope of the supporting line containing the segment. Given a family of graphs $\mathcal{G}$ and a drawing type $\mathcal{D}$ (for example planar drawings or outer 1-planar drawings), a set of slopes $\mathcal{S}$ is universal for $\langle\mathcal{G}, \mathcal{D}\rangle$, if every graph $G$ in $\mathcal{G}$ admits a drawing $\Gamma$ that respects the drawing type $\mathcal{D}$ and that only uses slopes in $\mathcal{S}$. In Section 3 we will define a universal set of slopes for planar straight-line drawings of outer 1-planar graphs with maximum degree $\Delta$. Similarly, in Section 4 we will define a universal set of slopes for outer 1-planar straight-line drawings of outer 1-planar graphs with maximum degree $\Delta$.

$S P Q R$-tree Decomposition. Let $G$ be a biconnected graph, then a separation pair is a pair of vertices whose removal disconnects $G$. A split pair is either a separation pair or a pair of adjacent vertices. A split component of a split pair $\{u, v\}$ is either an edge $(u, v)$ or a maximal subgraph $G_{u v} \subset G$ such that $\{u, v\}$ is not a split pair of $G_{u v}$. Vertices $\{u, v\}$ are the poles of $G_{u v}$. The $S P Q R$-tree $T$ of $G$ with respect to an edge $e$ is a rooted tree that describes 


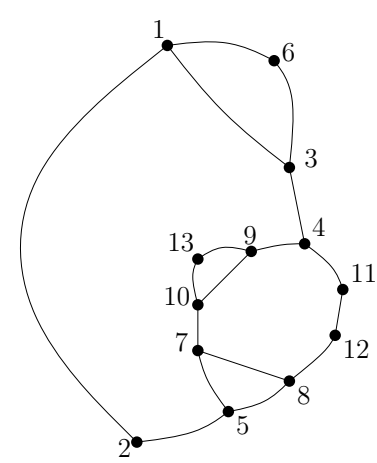

(a)

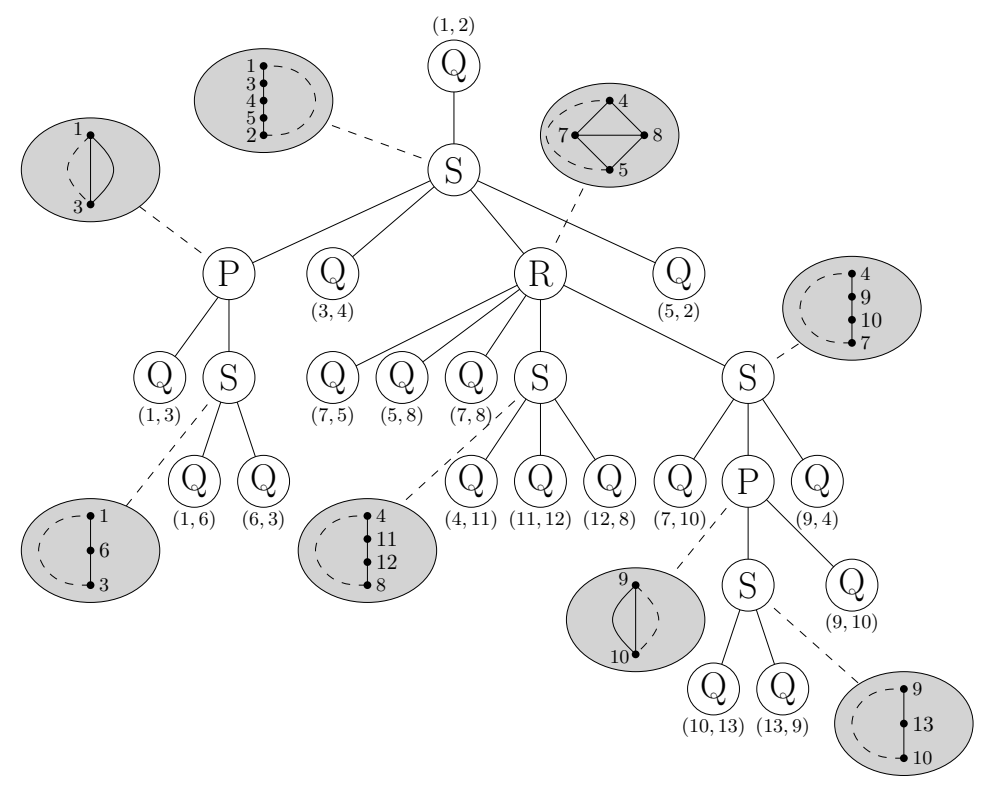

(b)

Figure 2: (a) A planar graph $G$; (b) The $S P Q R$-tree $T$ of $G$. For each node that is not a $Q$-node the skeleton is depicted in the gray balloons; for $Q$-nodes the corresponding edge is indicated. 
a recursive decomposition of $G$ induced by its split pairs. In what follows, we use the term nodes for the vertices of $T$, to distinguish them from the vertices of $G$. The nodes of $T$ are of four types $S, P, Q$, or $R$. Each node $\mu$ of $T$ has an associated biconnected multigraph called the skeleton of $\mu$, and denoted as $\sigma(\mu)$. The skeleton of $\mu$ contains a marked edge, called the reference edge. At each step, given the current split component $G^{*}$, its split pair $\{s, t\}$, and a node $\nu$ in $T$, the node $\mu$ of the tree corresponding to $G^{*}$ is introduced and attached to its parent vertex $\nu$, while the decomposition possibly recurses on some split component of $G^{*}$. At the beginning of the decomposition the parent of $\mu$ is a $Q$-node corresponding to the edge $e=(u, v), G^{*}=G \backslash e$, and $\{s, t\}=\{u, v\}$. In the recursive step, one of the following cases applies. See also Figure 2 .

- Base case: $G^{*}$ consists of a single edge between $s$ and $t$. Then, $\mu$ is a $Q$-node whose skeleton is $G^{*}$ itself plus the reference edge $(s, t)$.

- Parallel case: The split pair $\{s, t\}$ has split components $G_{1}, G_{2}, \ldots, G_{k}$ $(k \geq 2)$. Then, $\mu$ is a $P$-node whose skeleton is composed of $k+1$ parallel edges between $s$ and $t$, one for each split component $G_{i}$, plus the reference edge $(s, t)$. The decomposition recurses on each $G_{i}$ with $\mu$ as parent node.

- Series case: $G^{*}$ is not biconnected, and therefore it has at least one cut vertex (a vertex whose removal disconnects $G^{*}$ ). Then, $\mu$ is an $S$-node whose skeleton is defined as follows. Let $v_{1}, v_{2}, \ldots, v_{k-1}$, where $k \geq 2$, be the cut vertices of $G^{*}$. The skeleton of $\mu$ is a path $e_{1}, e_{2}, \ldots, e_{k}$, where $e_{i}=$ $\left(v_{i-1}, v_{i}\right), v_{0}=s$ and $v_{k}=t$, plus the reference edge $(s, t)$ which makes the path a cycle. The decomposition recurses on the split components corresponding to each $e_{1}, e_{2}, \ldots, e_{k}$ with $\mu$ as parent node.

- Rigid case: None of the other cases is applicable. A split pair $\left\{s^{\prime}, t^{\prime}\right\}$ is maximal with respect to $\{s, t\}$, if for every other split pair $\left\{s^{*}, t^{*}\right\}$, there is a split component that includes the vertices $s^{\prime}, t^{\prime}, s, t$. Let $\left\{s_{1}, t_{1}\right\}$, $\left\{s_{2}, t_{2}\right\}, \ldots,\left\{s_{k}, t_{k}\right\}$ be the maximal split pairs of $G^{*}$ with respect to $\{s, t\}(k \geq 1)$, and, for $i=1,2, \ldots, k$, let $G_{i}$ be the union of all the split components of $\left\{s_{i}, t_{i}\right\}$. Then $\mu$ is an $R$-node whose skeleton is obtained from $G^{*}$ by replacing each component $G_{i}$ with an edge between $s_{i}$ and $t_{i}$, plus the reference edge $(s, t)$. The decomposition recurses on each $G_{i}$ with $\mu$ as parent node.

$S P Q R$-trees of a graph $G$ with respect to different edges are the same if considered as unrooted trees. So computing an $S P Q R$-tree with respect to a different edge is equivalent to choose a different root for $T$.

Let $\mu$ be a node of $T$ and consider its skeleton $\sigma(\mu)$. Let $e_{1}, e_{2}, \ldots, e_{k}$ $(k \geq 1)$ be the edges of $\sigma(\mu)$ different from the reference edge $\left(s_{\mu}, t_{\mu}\right)$. Denote by $\nu_{i}(1 \leq i \leq k)$ the child of $\mu$ corresponding to the edge $e_{i}$. The frame of $\mu$ is the graph obtained from $\sigma(\mu)$ by removing the reference edge $\left(s_{\mu}, t_{\mu}\right)$. The pertinent graph of $\mu$, denoted by $G_{\mu}$, is a subgraph of $G$ defined recursively as follows. If $\mu$ is a $Q$-node, then $G_{\mu}$ coincides with the frame of $\mu$, i.e., it is a 


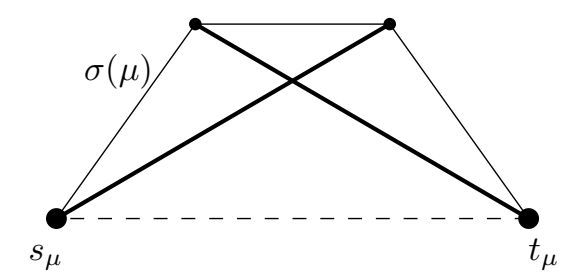

(a)

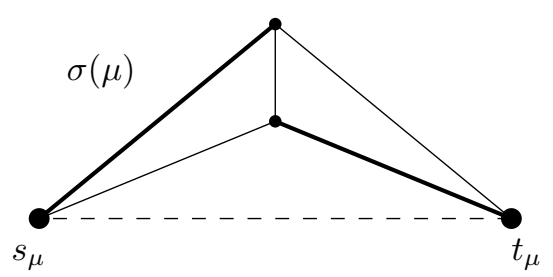

(b)

Figure 3: Skeleton $\sigma(\mu)$ of an $R$-node $\mu$. The dashed edge is the reference edge, while the two bold edges are the two edges of $G$ associated with two children of $\mu$ that are $Q$-nodes. (a) An outer 1-planar embedding of $\sigma(\mu)$; (b) a planar embedding of $\sigma(\mu)$.

single edge between the poles of $\mu$. If $\mu$ is an internal node, then $G_{\mu}$ is obtained from its frame by replacing each edge $e_{i}$ with the pertinent graph $G_{\nu_{i}}$ of $\nu_{i}$ (for $i=1,2, \ldots, k)$.

Structural Properties of Outer 1-planar Graphs. The structural properties of outer 1-planar graphs have been studied in [2, 14. Here we list a few of them that will be useful in Sections 3 and 4 .

Let $G$ be a biconnected outer 1-planar graph and let $T$ be its $S P Q R$-tree rooted at an arbitrary $Q$-node. Then the next property derives from Lemma 5 in [14 and defines the structure of the skeleton of the $R$-nodes of $T$ (see also Figure 3).

Property 1 Let $\mu$ be an $R$-node of $T$. Then:

(i) The skeleton $\sigma(\mu)$ is isomorphic to $K_{4}$.

(ii) Two children of $\mu$ are $Q$-nodes such that they do not share any pole.

We now give a structural property on the children of a $P$-node. Its proof is based on the following fact proved in [14]: Given a pair of vertices $u$ and $v$ in an outer 1-planar graph $G$, there can be at most five edge-disjoint paths connecting $u$ and $v$; also, if the number of paths is five, one of them is a single edge.

Property 2 There exists at most one $P$-node in $T$ with more than one $R$-node as a child. Also, if such a P-node exists, it is the child of the root of $T$ and it has exactly two children.

Proof: Assume $\xi$ is a $P$-node of $T$ having two $R$-nodes among the set of its children. We first prove that $\xi$ does not have any further child besides these two $R$-nodes, and then show that the parent of $\xi$ is the root of $T$.

Let $\mu_{1}$ and $\mu_{2}$ be two $R$-nodes whose parent is $\xi$ and let $s_{\xi}$ and $t_{\xi}$ be the poles of $\xi$. By Property 1$] \sigma\left(\mu_{1}\right)$ is isomorphic to $K_{4}$; hence, the pertinent graph $G_{\mu_{1}}$ contains two edge-disjoint paths connecting $s_{\xi}$ and $t_{\xi}$ and so does $G_{\mu_{2}}$. Note that each such path has at least two edges. Since the maximum number of 
edge-disjoint paths between the same pair of vertices in any outer 1-planar graph is at most five [14, and since the subgraph of $G$ represented by the reference edge $\left(s_{\xi}, t_{\xi}\right)$ contains at least one more edge-disjoint path connecting $s_{\xi}$ and $t_{\xi}$, it follows that $\xi$ cannot have a third child. Indeed, a third child would imply the existence of a sixth path between the two poles. Furthermore, as recalled above, one of the five paths must be an edge. It follows that the parent of $\xi$ is a $Q$-node. Since the root of $T$ is the only $Q$-node with a child, then the parent of $\xi$ is the root of $T$.

\section{The Planar Slope Number}

In this section we describe an algorithm, called BP-DrAwER, that computes a planar drawing of a biconnected outer 1-planar graph $G$ with maximum degree $\Delta$, using at most $4 \Delta^{2}-4 \Delta$ slopes. This result is then extended to simply connected graphs with a number of slopes equal to $4 \Delta^{2}+12 \Delta+8$.

A Universal Set of Slopes. We start by defining a universal set of slopes that are used by algorithm BP-DRAWER to draw in a planar way every biconnected outer 1-planar graph with maximum degree $\Delta$. Let $\theta=\frac{\pi}{4 \Delta}$ and observe that $0<\theta \leq \frac{\pi}{12}$ when $\Delta \geq 3$. Then denote by green slopes the set of slopes defined as $g_{i}=(i-1) \theta$, for $i=1,2, \ldots, 4 \Delta$. For each green slope $g_{i}$, we define $\Delta-1$ yellow slopes as $y_{i, j}=g_{i}+\arctan \left(\frac{\tan \left(g_{4} \Delta\right) \tan \left(g_{3}\right)}{\tan \left(g_{j}\right)}\right)$ with $j=3 \Delta, \ldots, 4 \Delta-2$. The reason for this choice will be clarified in the proof of Lemma 4. The union of the green and yellow slopes defines the universal set of slopes $\mathcal{T}_{\Delta}$. We observe that $g_{i}<y_{i, j}<g_{i+1}$, for each $1 \leq i<4 \Delta$ and $3 \Delta \leq j \leq 4 \Delta-2$. That is, $\tan \left(g_{4 \Delta}\right)=-\tan \left(g_{2}\right)=-\tan (\theta)$ (see Figure 4(a)). Also, for each $j=$ $3 \Delta, \ldots, 4 \Delta-2, \tan \left(g_{j}\right)$ is negative and larger in absolute value than $\tan \left(g_{3}\right)$ (which is positive). It follows that the argument of the arctangent is positive and strictly smaller than $\tan (\theta)$; since the arctangent function is monotonically increasing in the range $\left(-\frac{\pi}{2}, \frac{\pi}{2}\right)$, the term added to $g_{i}$ is strictly smaller than $\theta$, i.e, $y_{i, j}<g_{i+1}$. On the other hand, the argument of the arctangent is greater than 0 for every $j=3 \Delta, \ldots, 4 \Delta-2$ and thus $g_{i}<y_{i, j}$.

Algorithm Overview. Algorithm BP-Drawer takes as input a biconnected outer 1-planar graph $G$ with maximum degree $\Delta$ and returns a planar straightline drawing $\Gamma$ of $G$ that uses only slopes in $\mathcal{T}_{\Delta}$. Figure 5 shows a drawing computed by algorithm BP-Drawer. It first constructs the $S P Q R$-tree $T$ rooted at a $Q$-node $\rho$, and then draws $G$ by visiting $T$ bottom-up. At each step a node $\mu$ of $T$ different from the child of the root is processed and a drawing $\Gamma_{\mu}$ of its pertinent graph $G_{\mu}$ is computed. If $\mu$ is a $Q$-node, then its pertinent graph is an edge $\left(s_{\mu}, t_{\mu}\right)$ and is drawn as a horizontal segment of unit length. If $\mu$ is not a $Q$-node (i.e., is not a leaf), $\Gamma_{\mu}$ is computed by properly combining the already computed drawings of the pertinent graphs of the children of $\mu$. Let $s_{\mu}$ and $t_{\mu}$ be the poles of $\mu$. We denote by $\Delta\left(s_{\mu}\right)$ and $\Delta\left(t_{\mu}\right)$ the degree of $s_{\mu}$ and $t_{\mu}$ in $G_{\mu}$, respectively. Then for each drawing $\Gamma_{\mu}$ the algorithm maintains the following three invariants. 


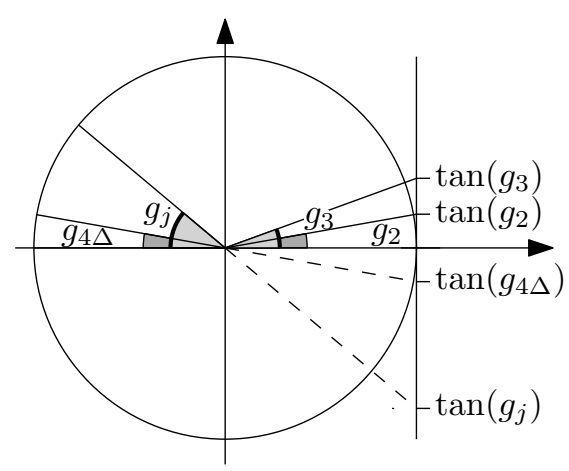

(a)

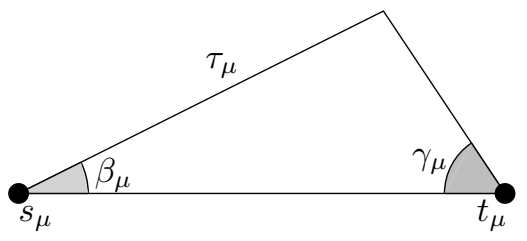

(b)

Figure 4: (a) Illustration of the green slopes. (b) Illustration of Invariant Ic

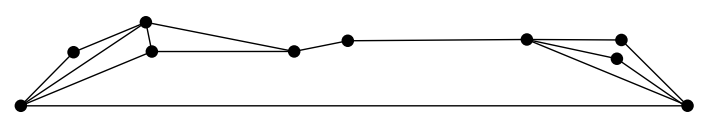

Figure 5: A planar drawing computed by algorithm BP-Drawer. The maximum degree of the input graph is $\Delta=4$.

Ia. $\Gamma_{\mu}$ is planar.

Ib. $\Gamma_{\mu}$ uses only slopes in $\mathcal{T}_{\Delta}$.

Ic. $\Gamma_{\mu}$ is contained in a triangle $\tau_{\mu}$ such that $s_{\mu}$ and $t_{\mu}$ are placed at the corners of its base. Also, $\beta_{\mu} \leq\left(\Delta\left(s_{\mu}\right)-1\right) \theta$ and $\gamma_{\mu} \leq\left(\Delta\left(t_{\mu}\right)-1\right) \theta$, where $\beta_{\mu}$ and $\gamma_{\mu}$ are the internal angles of $\tau_{\mu}$ at $s_{\mu}$ and $t_{\mu}$, respectively (see Figure $4(\mathrm{~b})$.

The root $\rho$ of $T$ and its unique child $\xi$ are handled in a special way. If $\mu$ is a $Q$-node, $G_{\mu}$ is an edge and its drawing is a horizontal segment that satisfies Invariants Ia, Ib, and Ic. About Invariant Ic, the triangle $\tau_{\mu}$ is, in this case, a degenerate triangle whose height is 0 . If $\mu$ is not a $Q$-node, $\Gamma_{\mu}$ is computed by combining the drawings $\Gamma_{\eta_{1}}, \Gamma_{\eta_{2}}, \ldots, \Gamma_{\eta_{k}}$ of the pertinent graphs $G_{\eta_{1}}, G_{\eta_{2}}, \ldots, G_{\eta_{k}}$ of the children $\eta_{1}, \eta_{2}, \ldots, \eta_{k}$ of $\mu$. To this aim, if necessary, the drawings $\Gamma_{\eta_{1}}, \Gamma_{\eta_{2}}, \ldots, \Gamma_{\eta_{k}}$ are manipulated by applying the following operations.

- The triangle $\tau_{\eta_{j}}(1 \leq j \leq k)$ can be arbitrarily scaled without modifying the slopes used in $\Gamma_{\eta_{j}}$. Observe that if $\eta_{j}$ is a $Q$-node, then $\tau_{\eta_{j}}$ is a segment, and the scaling operation only changes its length.

- The triangle $\tau_{\eta_{j}}(1 \leq j \leq k)$ can be rotated by an angle $c \cdot \theta$, with $c$ integer. The resulting drawing maintains invariant Ib. In fact, each green slope $g_{i}$, for $i=1,2, \ldots, 4 \Delta$, used in $\tau_{\eta_{j}}$ will be transformed into another green slope $g_{i+c}=(i-1+c) \theta=g_{i}+c \cdot \theta$, where $i+c$ is considered modulo $4 \Delta$. 

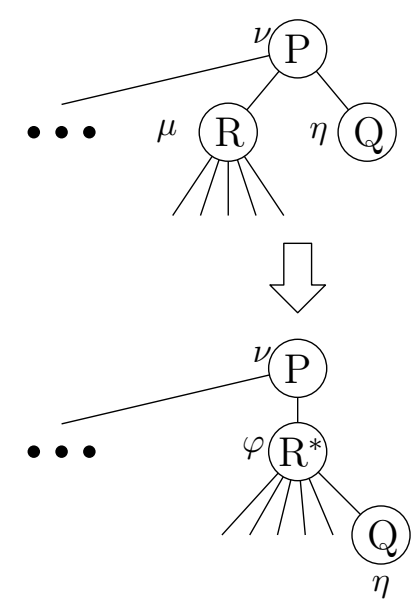

(a)
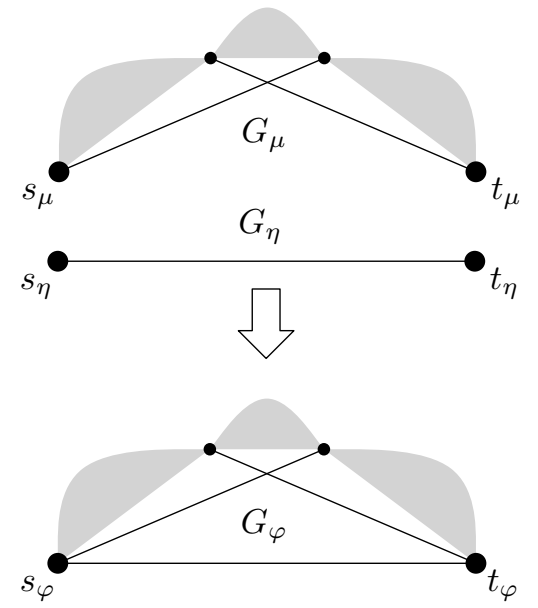

(b)

Figure 6: Illustration of an $R^{*}$-node: (a) Transformation of the $S P Q R$-tree; (b) Merging $G_{\mu}$ and $G_{\eta}$ into $G_{\phi}$.

Similarly, any yellow slope $y_{i, j}$ will be transformed into another yellow slope $y_{i+c, j}$.

- Finally, although the children of $\mu$ may share one or both the poles, we consider each pertinent graph to have its own copy of its poles. Then, given two drawings $\Gamma_{\eta_{i}}$ to $\Gamma_{\eta_{j}}$ (with $1 \leq i<j \leq k$ ) that share either two poles (this is always true when $\mu$ is a $P$-node) or one pole (this may happen when $\mu$ is either an $S$ - or $R$-node), we say that we attach $\Gamma_{\eta_{i}}$ to $\Gamma_{\eta_{j}}$ meaning that we make the points representing the shared poles to coincide.

Before describing how BP-DRAWER works in details, we need to distinguish between $R$-nodes whose poles are adjacent in $G$ and $R$-nodes whose poles are not adjacent in $G$. For this reason we introduce $R^{*}$-nodes. Let $\mu$ be an $R$-node; then if the poles $s_{\mu}$ and $t_{\mu}$ of $\mu$ are adjacent in $G$, the parent $\nu$ of $\mu$ is a $P$-node that has (at least) another child $\eta$ that is a $Q$-node (the edge associated with $\eta$ is $\left.\left(s_{\mu}, t_{\mu}\right)\right)$. We replace $\mu$ with a new node $\varphi$, that, for the sake of description, is called an $R^{*}$-node and we make $\eta$ a child of $\varphi$. Also, the children of $\mu$ become children of $\varphi$. If $\mu$ and $\eta$ were the only two children of $\nu$, then $\varphi$ also replaces $\nu$. The pertinent graph of $\varphi$ is $G_{\varphi}=G_{\mu} \cup G_{\eta}$, and the reference edge of $\varphi$ is $\left(s_{\mu}, t_{\mu}\right)$. See also Figure 6 .

The Drawing Algorithm. Algorithm BP-Drawer first computes the SPQRtree $T$ of $G$. Then, $R^{*}$-nodes are created if any. In the next lemmas we show how BP-Drawer computes a drawing $\Gamma_{\mu}$ of the pertinent graph $G_{\mu}$ of a node 


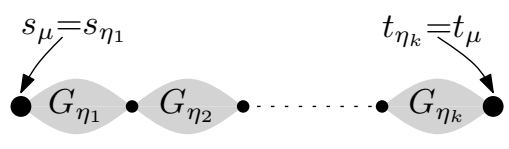

(a)

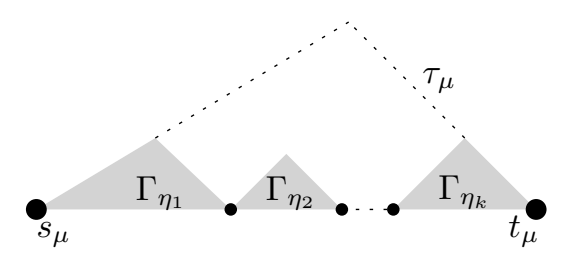

(b)

Figure 7: Illustration of Lemma1. (a) The pertinent graph $G_{\mu}$ of an $S$-node $\mu$. (b) The planar drawing of $G_{\mu}$.

$\mu$ of $T$, depending on the type of $\mu$. Recall that $\xi$ is the (only) child of the root $\rho$ of $T$ and that the leaves of $T$ are $Q$-nodes by definition.

Lemma 1 Let $\mu$ be an $S$-node different from $\xi$. Then $G_{\mu}$ admits a straight-line drawing $\Gamma_{\mu}$ that respects Invariants $\boldsymbol{I} \boldsymbol{a}, \boldsymbol{I} \boldsymbol{b}$, and $\boldsymbol{I} \boldsymbol{c}$.

Proof: Let $\eta_{1}, \eta_{2}, \ldots, \eta_{k}$ be the $k \geq 2$ children of $\mu$ in $T$. In order to construct $\Gamma_{\mu}$, the drawings $\Gamma_{\eta_{1}}, \Gamma_{\eta_{2}}, \ldots, \Gamma_{\eta_{k}}$ of the pertinent graphs of $\eta_{1}, \eta_{2}, \ldots, \eta_{k}$ are combined as follows, see also Figure 7. If $k>2$, then, in order to satisfy Invariant $\mathbf{I b}$, we need that the height of $\tau_{\eta_{i}}$ is less than the minimum between the height of $\tau_{\eta_{1}}$ and the height of $\tau_{\eta_{k}}$. To this aim, BP-Drawer scales down $\Gamma_{\eta_{i}}$, for $i=2, \ldots, k-1$, if necessary. Then, $\Gamma_{\eta_{1}}, \Gamma_{\eta_{2}}, \ldots, \Gamma_{\eta_{k}}$ are attached to each other so that the bases of the triangles $\tau_{\eta_{1}}, \tau_{\eta_{2}}, \ldots, \tau_{\eta_{k}}$ are all contained in the same horizontal straight line, and such that all the vertices of $G_{\mu}$ are above or on the horizontal segment $\overline{s_{\mu} t_{\mu}}$. Invariant Ia holds by construction because we combined the drawings in such a way that they do not intersect each other (except at common vertices). Invariant $\mathbf{I b}$ holds since the slopes of $\Gamma_{\eta_{i}}$, for $i=1, \ldots, k$, have not been changed. Invariant Ic holds because it holds for $\Gamma_{\eta_{1}}$ and $\Gamma_{\eta_{k}}$ and all triangles $\tau_{\eta_{i}}$ (for $i=1, \ldots, k$ ) have a height smaller than that of $\tau_{\eta_{1}}$ and $\tau_{\eta_{k}}$ (due to the scaling).

In order to prove the next lemma, we introduce an additional operation, denoted by $\operatorname{bend}\left(\Gamma_{\mu}, \beta_{\mu}^{*}, \gamma_{\mu}^{*}\right)$, that takes as input the drawing $\Gamma_{\mu}$ of an $S$-node $\mu$ (computed as shown in the proof of Lemma 1) together with two angles $\beta_{\mu}^{*}$ and $\gamma_{\mu}^{*}$, and transforms $\Gamma_{\mu}$ as follows. Let $\eta_{1}, \eta_{2}, \ldots, \eta_{k}$, be the $k \geq 2$ children of $\mu$ in $T$ and consider the drawings $\Gamma_{\eta_{1}}, \Gamma_{\eta_{2}}, \ldots, \Gamma_{\eta_{k}}$, of their pertinent graphs. $\Gamma_{\mu}$ is first rotated so that the segment $s_{\mu} t_{\mu}$ is contained in the line with slope $\beta_{\mu}^{*}$ that passes through $s_{\mu}$. Next, the subdrawing $\Gamma_{\eta_{k}}$ of $\Gamma_{\mu}$ is rotated so that the segment $\overline{s_{\eta_{k}} t_{\eta_{k}}}$ is contained in the line with slope $\pi-\gamma_{\mu}^{*}$ that passes through $t_{\eta_{k}}=t_{\mu}$. Finally, if necessary, $\Gamma_{\eta_{k}}$ is scaled, so that $s_{\mu}$ and $t_{\mu}$ are horizontally aligned. See also Figure 8 for an illustration.

Lemma 2 Let $\mu$ be a P-node different from $\xi$. Then $G_{\mu}$ admits a straight-line drawing $\Gamma_{\mu}$ that respects Invariants $\boldsymbol{I} \boldsymbol{a}, \boldsymbol{I} \boldsymbol{b}$, and $\boldsymbol{I} \boldsymbol{c}$. 


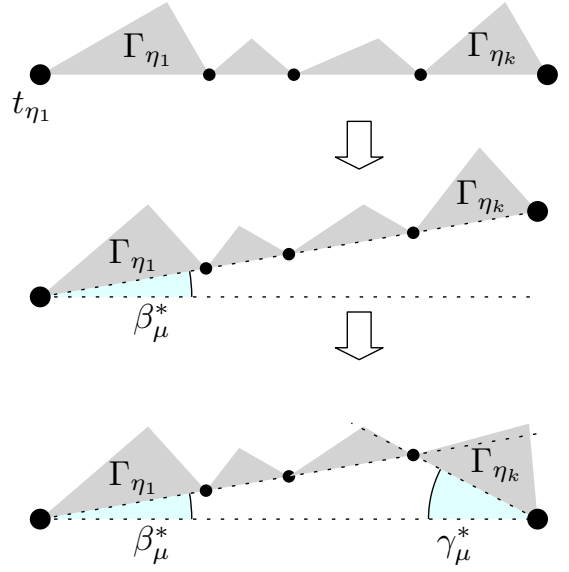

Figure 8: Illustration of the $\operatorname{bend}\left(\Gamma_{\mu}, \beta_{\mu}^{*}, \gamma_{\mu}^{*}\right)$ operation.

Proof: By Property 2, since $\mu$ is different from $\xi, \mu$ has at most one $R$-node among its children. Moreover, by the definition of the $S P Q R$-tree, it cannot have a $P$-node as a child. Thus, the children of $\mu$ are all $S$-nodes, except for at most one child, which can be an $R$-node, a $Q$-node or an $R^{*}$-node. Denote by $\eta_{1}, \eta_{2}, \ldots, \eta_{k}$, the $k \geq 2$ children of $\mu$, and let $\eta_{1}$ be the child of $\mu$ that is not an $S$-node, if it exists. Then in any case, $\eta_{2}, \eta_{3}, \ldots, \eta_{k}$ are all $S$-nodes. We apply the following operations: $\operatorname{bend}\left(\Gamma_{\eta_{i}}, \beta_{\eta_{i}}^{*}, \gamma_{\eta_{i}}^{*}\right)$, for $i=2,3, \ldots, k$, where the angles $\beta_{\eta_{i}}^{*}, \gamma_{\eta_{i}}^{*}$ are computed as follows. For $i=2$ we have $\beta_{\eta_{2}}^{*}=\beta_{\eta_{1}}+\theta$, $\gamma_{\eta_{2}}^{*}=\gamma_{\eta_{1}}+\theta$, while for $i=3,4, \ldots, k$ we have $\beta_{\eta_{i}}^{*}=\beta_{\eta_{i-1}}^{*}+\beta_{\eta_{i-1}}+\theta, \gamma_{\eta_{i}}^{*}=$ $\gamma_{\eta_{i-1}}^{*}+\gamma_{\eta_{i-1}}+\theta$. Then we attach $\Gamma_{\eta_{1}}, \Gamma_{\eta_{2}}, \ldots, \Gamma_{\eta_{k}}$ to each other (scaling some of them if necessary). See also Figure 9 for an illustration.

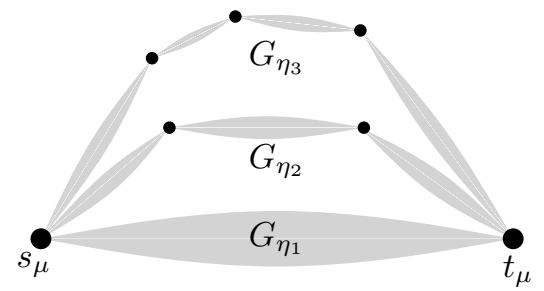

(a)

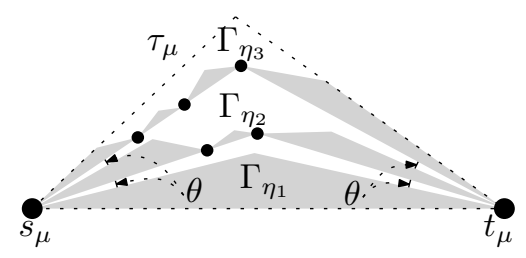

(b)

Figure 9: Illustration of Lemma 2. (a) The pertinent graph $G_{\mu}$ of an $P$-node $\mu$. (b) The planar drawing of $G_{\mu}$.

Invariants Ia and $\mathbf{I b}$ hold by construction, since we combined the drawings in such a way that they do not intersect each other (except at common vertices) and are rotated by angles that are integer multiples of $\theta$. Consider now Invariant Ic. By construction, $\Gamma_{\mu}$ is contained in a triangle $\tau_{\mu}$ such that $s_{\mu}$ and $t_{\mu}$ are placed 


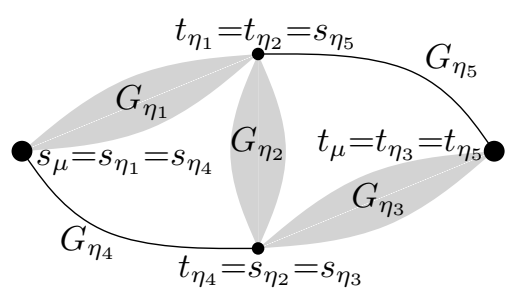

(a)

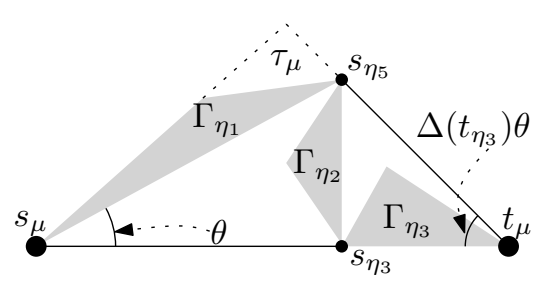

(b)

Figure 10: Illustration of Lemma 3 (a) The pertinent graph $G_{\mu}$ of an $R$-node $\mu$. (b) The planar drawing of $G_{\mu}$.

at the corners of its base. Also, we have that $\Delta\left(s_{\mu}\right)=\sum_{i=1}^{k} \Delta\left(s_{\eta_{i}}\right)$ and $\Delta\left(t_{\mu}\right)=$ $\sum_{i=1}^{k} \Delta\left(t_{\eta_{i}}\right)$. By construction, $\beta_{\mu}=\sum_{i=1}^{k} \beta_{\eta_{i}}+(k-1) \theta \leq \sum_{i=1}^{k}\left(\Delta\left(s_{\eta_{i}}\right)-1\right) \theta+$ $(k-1) \theta=\Delta\left(s_{\mu}\right) \theta-k \theta+(k-1) \theta=\left(\Delta\left(s_{\mu}\right)-1\right) \theta$. Similarly, $\gamma_{\mu}=\sum_{i=1}^{k} \gamma_{\eta_{i}}+$ $(k-1) \theta \leq \sum_{i=1}^{k}\left(\Delta\left(t_{\eta_{i}}\right)-1\right) \theta+(k-1) \theta=\Delta\left(t_{\mu}\right) \theta-k \theta+(k-1) \theta=\left(\Delta\left(t_{\mu}\right)-1\right) \theta$. Hence, Invariant Ic holds.

Lemma 3 Let $\mu$ be an $R$-node different from $\xi$. Then $G_{\mu}$ admits a straight-line drawing $\Gamma_{\mu}$ that respects Invariants $\boldsymbol{I} \boldsymbol{a}, \boldsymbol{I} \boldsymbol{b}$, and $\boldsymbol{I} \boldsymbol{c}$.

Proof: By Property 1, the skeleton $\sigma(\mu)$ of $\mu$ is isomorphic to $K_{4}$ and at least two children of $\mu$ are $Q$-nodes. Also, the two edges corresponding to these $Q$-nodes do not share an end vertex and each one of them is incident to a distinct pole of $\mu$. Let $\eta_{1}, \eta_{2}, \eta_{3}, \eta_{4}$, and $\eta_{5}$ be the children of $\mu$; then we assume that $\eta_{4}$ and $\eta_{5}$ are two $Q$-nodes that do not share a pole. Also, we assume $s_{\mu}=s_{\eta_{1}}=s_{\eta_{4}}, t_{\mu}=t_{\eta_{3}}=t_{\eta_{5}}, t_{\eta_{1}}=t_{\eta_{2}}=s_{\eta_{5}}$, and $t_{\eta_{4}}=s_{\eta_{2}}=s_{\eta_{3}}$. See also Figure 10(a) for an illustration.

We construct a drawing of $G_{\mu}$ as follows, see also Figure 10(b). We draw the edge associated with $\eta_{5}$ as a segment whose slope is the green slope ( $4 \Delta-$ $\left.\Delta\left(t_{\eta_{3}}\right)\right) \theta$ and whose length is such that $s_{\eta_{5}}$ is vertically aligned with $s_{\eta_{3}}$. We rotate $\Gamma_{\eta_{2}}$ so that the segment $\overline{s_{\eta_{2}} t_{\eta_{2}}}$ uses the green slope $g_{2 \Delta+1}=\frac{\pi}{2}$. We then attach $\Gamma_{\eta_{2}}, \Gamma_{\eta_{3}}$, and $\Gamma_{\eta_{5}}$ to each other (scaling some of them if necessary). We draw the edge corresponding to $\eta_{4}$ with the horizontal slope $g_{1}$, and stretch it so that $s_{\eta_{4}}=s_{\mu}$ belongs to the line with slope $g_{2}$ passing through $s_{\eta_{5}}$. We rotate $\Gamma_{\eta_{1}}$ so that the segment $\overline{s_{\eta_{1}} t_{\eta_{1}}}$ uses the green slope $g_{2}$. We then attach $\Gamma_{\eta_{1}}, \Gamma_{\eta_{5}}$, and $\Gamma_{\eta_{4}}$ (scaling some of them if necessary). Invariant Ib holds because $\Gamma_{\eta_{1}}, \Gamma_{\eta_{2}}, \Gamma_{\eta_{3}}, \Gamma_{\eta_{4}}$, and $\Gamma_{\eta_{5}}$ are rotated by angles that are integer multiples of $\theta$. Invariant Ia holds because the drawings are combined so that they do not intersect each other except at common endpoints. To see this fact, we show that $\Gamma_{\eta_{2}}$ is completely contained inside the triangle $\tau$ defined by the three vertices $s_{\mu}, s_{\eta_{3}}$, and $s_{\eta_{5}}$ (except for the segment $\overline{s_{\eta_{3}} s_{\eta_{5}}}$ that $\Gamma_{\eta_{2}}$ shares with $\tau$ ). The angle inside $\tau$ at $s_{\eta_{3}}$ is $\frac{\pi}{2}$, while the angle inside $\tau$ at $s_{\eta_{5}}$ is at least $\frac{\pi}{4}$ (because the angle inside $\tau$ at $s_{\mu}$ is $\theta<\frac{\pi}{4}$ ). On the other hand, $\beta_{\eta_{2}} \leq\left(\Delta\left(s_{\eta_{2}}\right)-1\right) \theta \leq(\Delta-3) \theta<\frac{\pi}{4}$, and $\gamma_{\eta_{2}} \leq\left(\Delta\left(t_{\eta_{2}}\right)-1\right) \theta \leq(\Delta-3) \theta<\frac{\pi}{4}$. 


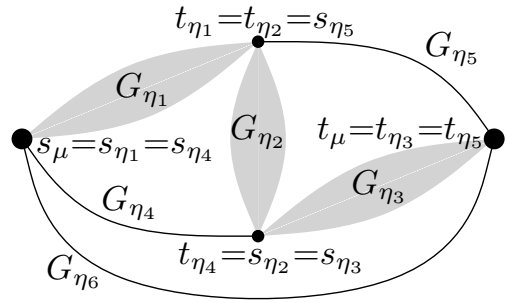

(a)

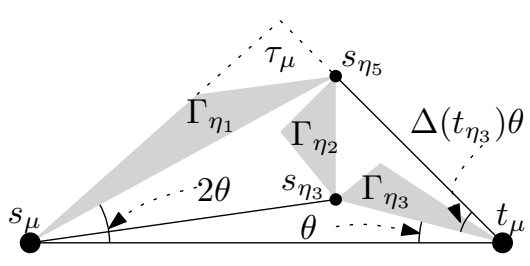

(b)

Figure 11: Illustration of Lemma 4 (a) The pertinent graph $G_{\mu}$ of an $R^{*}$-node $\mu$. (b) The planar drawing of $G_{\mu}$.

Thus, the triangle $\tau_{\eta_{2}}$ is completely inside $\tau$ except for the vertical side shared by the two triangles. This implies that $\Gamma_{\eta_{2}}$ does not intersect $\Gamma_{\eta_{1}}$ and $\Gamma_{\eta_{3}}$ (except at common endpoints). Concerning Invariant Ic, we have that $\Delta\left(s_{\mu}\right)=\Delta\left(s_{\eta_{1}}\right)+1$, and $\Delta\left(t_{\mu}\right)=\Delta\left(t_{\eta_{3}}\right)+1$. Moreover, $\beta_{\mu}=\beta_{\eta_{1}}+\theta \leq\left(\Delta\left(s_{\eta_{1}}\right)-1\right) \theta+\theta=\Delta\left(s_{\eta_{1}}\right) \theta=$ $\left(\Delta\left(s_{\mu}\right)-1\right) \theta$. Finally, $\gamma_{\mu}=\gamma_{\eta_{3}}+\theta \leq\left(\Delta\left(t_{\eta_{3}}\right)-1\right) \theta+\theta=\Delta\left(t_{\eta_{3}}\right) \theta=\left(\Delta\left(t_{\mu}\right)-1\right) \theta$.

Lemma 4 Let $\mu$ be an $R^{*}$-node different from $\xi$. Then $G_{\mu}$ admits a straightline drawing $\Gamma_{\mu}$ that respects Invariants $\boldsymbol{I} \boldsymbol{a}, \boldsymbol{I} \boldsymbol{b}$, and $\boldsymbol{I} \boldsymbol{c}$.

Proof: Since $\mu$ is an $R^{*}$-node, it is obtained by merging an $R$-node $\mu^{\prime}$ and a $Q$-node representing the edge $\left(s_{\mu^{\prime}}, t_{\mu^{\prime}}\right)$. Following the same notation used in Lemma 3 , let $\eta_{1}, \eta_{2}, \eta_{3}, \eta_{4}$, and $\eta_{5}$ be the children of $\mu^{\prime}$, as in Figure 11(a). Also, $\mu$ has a sixth child $\eta_{6}$ that is a $Q$-node corresponding to the edge $\left(s_{\mu}, t_{\mu}\right)$. We construct a drawing of $G_{\mu}$ in a similar way as in the proof of Lemma 3 (see Figure 11(b) . The main difference is that we need to rotate $\Gamma_{\eta_{3}}$ and $\Gamma_{\eta_{4}}$. In the $R$-node case, the horizontal line through $s_{\mu}$ and $t_{\mu}$ contained the segments $\overline{s_{\eta_{3}} t_{\eta_{3}}}$ and $\overline{s_{\eta_{4}} t_{\eta_{4}}}$, while in the present case it has to host the edge $\left(s_{\mu}, t_{\mu}\right)$. As a consequence $\Gamma_{\eta_{3}}$ and $\Gamma_{\eta_{4}}$ have to be rotated. We rotate $\Gamma_{\eta_{3}}$ so that the segment $\overline{s_{\eta_{3}} t_{\eta_{3}}}$ uses the green slope $g_{4 \Delta}$. Then, we draw the edge associated with $\eta_{5}$ as a segment whose slope is the green slope $g_{j}$, with $j=\left(4 \Delta-\Delta\left(t_{\eta_{3}}\right)\right)$, and whose length is such that $s_{\eta_{5}}$ is vertically aligned with $s_{\eta_{3}}$. In the same way as for $R$-nodes, we rotate $\Gamma_{\eta_{2}}$ so that the segment $\overline{s_{\eta_{2}} t_{\eta_{2}}}$ uses the green slope $g_{2 \Delta+1}=\frac{\pi}{2}$. We then attach $\Gamma_{\eta_{2}}, \Gamma_{\eta_{3}}$, and $\Gamma_{\eta_{5}}$ (scaling some of them if necessary). We draw the edge corresponding to $\eta_{6}$ with the horizontal slope $g_{1}$, and stretch it so that $s_{\eta_{6}}=s_{\mu}$ belongs to the line with slope $g_{3}$ passing through $s_{\eta_{5}}$. We now rotate $\Gamma_{\eta_{1}}$ so that the segment $\overline{s_{\eta_{1}} t_{\eta_{1}}}$ uses the green slope $g_{3}$ (unlike in the $R$-node case where we used the slope $g_{2}$ ) and attach it to $\Gamma_{\eta_{5}}$ and $\Gamma_{\eta_{6}}$. Finally, the edge corresponding to $\eta_{4}$ is drawn as the segment $\overline{s_{\mu} s_{\eta_{3}}}$. Invariant Ia holds because the drawings $\Gamma_{\eta_{1}}, \Gamma_{\eta_{2}}, \Gamma_{\eta_{3}}, \Gamma_{\eta_{4}}, \Gamma_{\eta_{5}}$, and $\Gamma_{\eta_{6}}$ do not intersect each other except at common endpoints. To see this fact, we show that $\Gamma_{\eta_{2}}$ is completely contained inside the triangle $\tau$ defined by the three vertices $s_{\mu}, s_{\eta_{3}}$, and $s_{\eta_{5}}$ (except for the segment $\overline{s_{\eta_{3}} s_{\eta_{5}}}$ that $\Gamma_{\eta_{2}}$ shares with $\tau$ ). The angle inside $\tau$ at $s_{\eta_{3}}$ is $\frac{\pi}{2}+\phi$, where $\phi$ is the slope of the edge corresponding 


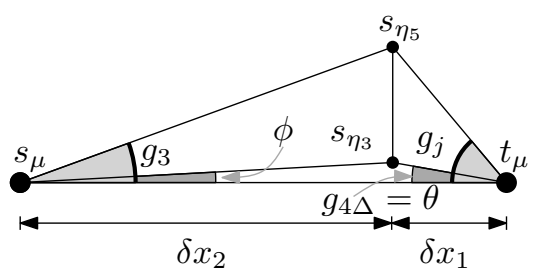

Figure 12: Illustration for the proof of Lemma 4 .

to $\eta_{4}$. The angle inside $\tau$ at $s_{\mu}$ is equal to $2 \theta-\phi$. Then, the angle inside $\tau$ at $s_{\eta_{5}}$ is $\pi-\frac{\pi}{2}-\phi-2 \theta+\phi=\frac{\pi}{2}-2 \theta$, which is at least $\frac{\pi}{4}$ because $2 \theta<\frac{\pi}{4}$. Since $\beta_{\eta_{2}}<\frac{\pi}{4}$ and $\gamma_{\eta_{2}}<\frac{\pi}{4}$, the triangle $\tau_{\eta_{2}}$ is completely inside $\tau$ except for the vertical side shared by the two triangles. This implies that $\Gamma_{\eta_{2}}$ does not intersect $\Gamma_{\eta_{1}}$ and $\Gamma_{\eta_{3}}$ (except at common endpoints). Concerning Invariant $\mathbf{I b}$, we observe that $\Gamma_{\eta_{1}}, \Gamma_{\eta_{2}}, \Gamma_{\eta_{3}}$, and $\Gamma_{\eta_{5}}$ are rotated by an angle that is a multiple of $\theta$ and therefore $\mathbf{I b}$ holds by construction for each of them. We now show that the slope $\phi$ of the edge corresponding to $\eta_{4}$ is in fact either a green slope or a yellow one (see Figure 12). Let $\delta x_{1}$ be the horizontal distance between $s_{\eta_{3}}$ and $t_{\mu}$ and let $\delta x_{2}$ be the horizontal distance between $s_{\mu}$ and $s_{\eta_{3}}$. Then, by applying some trigonometry we have:

$$
-\delta x_{1} \tan \left(g_{4 \Delta}\right)=\delta x_{2} \tan (\phi)
$$

and:

$$
-\delta x_{1} \tan \left(g_{j}\right)=\delta x_{2} \tan \left(g_{3}\right)
$$

where $g_{j}$ is the slope of the segment representing the edge corresponding to $\eta_{5}$ (and therefore $j=4 \Delta-\Delta\left(t_{\eta_{3}}\right)$ ). From the two previous equations we obtain:

$$
\tan (\phi)=\frac{\tan \left(g_{4 \Delta}\right) \tan \left(g_{3}\right)}{\tan \left(g_{j}\right)}
$$

Note that $1 \leq \Delta\left(t_{\eta_{3}}\right) \leq \Delta$ and therefore $3 \Delta \leq j \leq 4 \Delta-1$. If $j=4 \Delta-1$, then $\tan \left(g_{3}\right)=-\tan \left(g_{j}\right)$ and $\tan (\phi)=-\tan \left(g_{4 \Delta}\right)=\tan \left(g_{2}\right)$, hence $\phi=g_{2}$, i.e., $\phi$ is a green slope. Otherwise $\phi=\arctan \left(\frac{\tan \left(g_{4} \Delta\right) \tan \left(g_{3}\right)}{\tan \left(g_{j}\right)}\right)$ and therefore $\phi$ is the yellow slope $y_{1, j}$ (recall that $g_{1}=0$ ). Concerning Invariant Ic, we have that $\Delta\left(s_{\mu}\right)=\Delta\left(s_{\eta_{1}}\right)+2$ and $\Delta\left(t_{\mu}\right)=\Delta\left(t_{\eta_{3}}\right)+2$. Moreover, $\beta_{\mu}=\beta_{\eta_{1}}+2 \theta \leq$ $\left(\Delta\left(s_{\eta_{1}}\right)-1\right) \theta+2 \theta=\left(\Delta\left(s_{\mu}\right)-1\right) \theta$. Finally, $\gamma_{\mu}=\gamma_{\eta_{3}}+2 \theta \leq\left(\Delta\left(t_{\eta_{3}}\right)-1\right) \theta+2 \theta=$ $\left(\Delta\left(t_{\mu}\right)-1\right) \theta$.

In the next lemma we process the root of $T$ and its child. Since after that there are no other nodes to be processed, Invariant Ic is not needed anymore.

Lemma 5 Let $\rho$ be the root of $T$ and let $\xi$ be its unique child. Then $G=G_{\rho} \cup G_{\xi}$ admits a straight-line drawing $\Gamma$ that respects Invariants $\boldsymbol{I} \boldsymbol{a}$ and $\boldsymbol{I} \boldsymbol{b}$. 


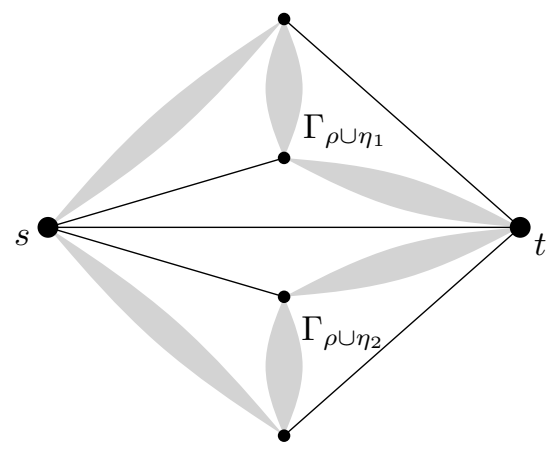

Figure 13: Illustration for the proof of Lemma 5

Proof: Denote by $(s, t)$ the edge associated with $\rho$. Observe that $s=s_{\xi}$ and $t=t_{\xi}$. If $\xi$ is an $R$-node, then $G=G_{\rho} \cup G_{\xi}$ corresponds to the pertinent graph of an $R^{*}$-node, thus the drawing $\Gamma_{\mu}$ of $G_{\mu}$ can be computed with the same technique described in the proof of Lemma 4 for $R^{*}$-nodes. Thus, invariants Ia and $\mathbf{I b}$ hold. If $\xi$ is an $S$-node, we first compute a drawing of $G_{\xi}$ according to the technique of Lemma 1 , then the operation $\operatorname{bend}\left(\Gamma_{\xi}, \theta, \theta\right)$ is performed and finally the edge $(s, t)$ is added by using the horizontal green slope $g_{1}$. Also in this case invariants $\mathbf{I a}$ and $\mathbf{I b}$ hold. If $\xi$ is a $P$-node, we need to distinguish between two cases. Either $\xi$ has two $R$-nodes as children or not. If $\xi$ has at most one child that is an $R$-node, then $G=G_{\rho} \cup G_{\xi}$ can be drawn with the same technique described in the proof of Lemma 2 , for which invariants Ia and $\mathbf{I b}$ hold. If instead $\xi$ has exactly two $R$-nodes as children, denote these two children as $\eta_{1}$ and $\eta_{2}$. Observe that $G_{\rho} \cup G_{\eta_{1}}$ corresponds to the pertinent graph of an $R^{*}$-node, thus it can be drawn with the same technique described in the proof of Lemma 4 for $R^{*}$-nodes. Similarly for $G_{\rho} \cup G_{\eta_{2}}$. The two drawings can be attached by flipping (and possibly scaling) one of them as in Figure 13. Also in this case invariants Ia and Ib hold.

Lemma 6 Let $G$ be a biconnected outer 1-planar graph with $n$ vertices and with maximum degree $\Delta$. Then $G$ admits a planar straight-line drawing with at most $4 \Delta^{2}-4 \Delta$ slopes. Also, this drawing can be computed in $O(n)$ time in the real RAM model of computation.

Proof: By Lemmas 1, 2, 3, 4, and 5, $G$ has a planar straight-line drawing with at most $4 \Delta^{2}-4 \Delta$ slopes. Concerning the time complexity, we recall that the $S P Q R$-tree $T$ of $G$ can be computed in $O(n)$ time [7, and the $R^{*}$-nodes can be created (if any) in $O(n)$ time, as $T$ has $O(n)$ nodes.

In order to achieve linear time complexity, we implement the drawing computation phase of BP-DrAWER so that it works in two phases. In the first phase we perform a bottom-up visit of $T$ and for each node $\mu$, we compute a drawing of the frame of $\mu$ for $S$-nodes, $R$-nodes, and $R^{*}$-nodes or we combine the drawings of the frames of the children of $\mu$ for $P$-nodes. At the end of 


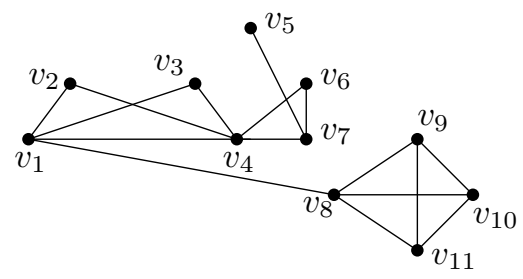

(a)

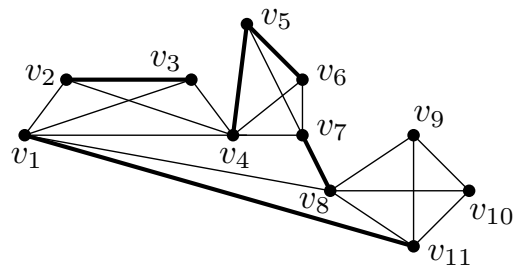

(b)

Figure 14: Illustration of the technique to biconnect a connected outer 1-plane graph. The numbering of the vertices corresponds to a possible order obtained walking clockwise along the border of the outer face. In (a) a connected outer 1-plane graph $G$ is shown. In (b) the bold edges are those that have been inserted to make $G$ biconnected.

this phase, each node of $T$ is associated with a drawing of its frame (for $S$ nodes, $R$-nodes, and $R^{*}$-nodes) or with a drawing of the union of the frames of its children (for $P$-nodes). Let $n_{\mu}^{*}$ be the number of vertices in the drawing associated with each node $\mu$. This drawing can be computed in $O\left(n_{\mu}^{*}\right)$ time. The total number of vertices and edges of all the skeletons of $T$ is $O(n)$ [7. Thus, $\sum_{\mu \in T} O\left(n_{\mu}^{*}\right)=O(n)$ and the first phase can be executed in $O(n)$ time. In the second phase we perform a top-down visit of $T$ and compute the final coordinates of each vertex of $G$, thus combining all the drawings computed in the first phase. Once the coordinates of the vertices of the drawing associated with $\mu$ have been fixed, we can fix the coordinates of the vertices of the drawings associated with its children (or its grand-children in the case of $P$-nodes). Again, since $\sum_{\mu \in T} O\left(n_{\mu}^{*}\right)=O(n)$, the time complexity of this phase is $O(n)$. We remark that in the real RAM model of computation we can store arbitrary real numbers and we can compute rational functions over reals at unit cost (see, e.g., 24]).

Extension to Connected Graphs. We now describe how to handle simply connected graphs, i.e., graphs that are connected but not biconnected. It is known that a simply connected, outerplane graph (with a given outerplanar embedding) can be modified into a biconnected outerplane graph by adding edges 8 . This technique can be directly applied also to outer 1-plane graphs. More specifically, let $G$ be a simply connected outer 1-planar graph. Compute an outer 1-planar embedding of $G$ and consider the circular order of the vertices of $G$ obtained by walking clockwise along the border of the outer face of $G$ (starting from an arbitrary vertex). Observe that a vertex may be visited more than once (if it is a cut vertex), however we consider it in the ordering only the first time that we visit it. Then for each pair of consecutive vertices $u$ and $v$ in this order, such that $u$ and $v$ are not connected by an edge, we add the edge $(u, v)$. At the end of this process the resulting graph is still outer 1-planar, as the only edges that we added are between vertices that appear consecutively along the boundary of the outer face, and it is biconnected, as 
there exists a Hamiltonian cycle that passes through all the vertices. Moreover, let $\Delta$ be the maximum degree of the connected graph, then the maximum degree of the resulting biconnected graph is at most $\Delta+2$, since each vertex has one predecessor and one successor in the visit, and thus no more than two edges can be added to each vertex. Figure 14 shows an illustration of this technique. By applying BP-Drawer to the resulting biconnected graph and removing the inserted edges we obtain the following result.

Theorem 1 Let $G$ be an outer 1-planar graph with $n$ vertices and with maximum degree $\Delta$. Then $G$ admits a planar straight-line drawing with at most $4 \Delta^{2}+12 \Delta+8$ slopes. Also, this drawing can be computed in $O(n)$ time in the real RAM model of computation.

\section{The Outer 1-planar Slope Number}

In this section we present a second algorithm, called BO1P-DrAWER, that takes as input a biconnected outer 1-planar graph $G$ with maximum degree $\Delta$, and returns an outer 1-planar straight-line drawing $\Gamma$ of $G$ with at most $6 \Delta$ slopes. This result is then extended to simply connected graphs with a number of slopes equal to $6 \Delta+12$.

Properties of Outer 1-planar Embeddings. We now refine the properties introduced in Section 2 and introduce new properties that hold in the fixed outer 1-planar embedding setting and that follow from the results in 14.

We consider a biconnected outer 1-plane graph $G$ (i.e., an embedded graph) and its $S P Q R$-tree $T$. Let $\mu$ be a node of $T$, let $G_{\mu}$ be its pertinent graph and let $s_{\mu}, t_{\mu}$ be its poles. Then the augmented pertinent graph $G_{\mu}^{+}=G_{\mu} \cup\left(s_{\mu}, t_{\mu}\right)$ of $\mu$ is the graph obtained by adding the reference edge $\left(s_{\mu}, t_{\mu}\right)$ of $\mu$ to $G_{\mu}$. Notice that $G_{\mu}$ and $G_{\mu}^{+}=G_{\mu} \cup\left(s_{\mu}, t_{\mu}\right)$ are outer 1-plane graphs with the embeddings inherited from the embedding of $G$. We denote by $\rho$ the root of $T$, and by $\xi$ its (only) child.

The next property derives from Lemma 5 in [14, see also Figure 16(a), By Property 1 the skeleton of an $R$-node $\mu$ is isomorphic to $K_{4}$. The following property describes the crossings in an outer 1-planar embedding of $\sigma(\mu)$.

Property 3 Let $\mu$ be an R-node of T. Then, in any outer 1-planar embedding the skeleton $\sigma(\mu)$ has one crossing between two edges associated with two children of $\mu$ that are $Q$-nodes.

In order to handle $P$-nodes, we now define a special kind of $S$-nodes, similarly as done in [14].

Definition 1 Let $\mu$ be an $S$-node of $T$. Let $\eta$ be the unique child of $\mu$ having $s_{\mu}$ as a pole, and let $\eta^{\prime}$ be the unique child of $\mu$ having $t_{\mu}$ as a pole. Then node $\mu$ has a tail at $s_{\mu}\left(t_{\mu}\right)$, if $\eta\left(\eta^{\prime}\right)$ is a $Q$-node.

A schematic representation of an $S$-node with a tail at $s_{\mu}$ is depicted in Figure 15. The next property derives from Lemma 6 in [14, see also Figures $16(\mathrm{~b})$ 16(d). 


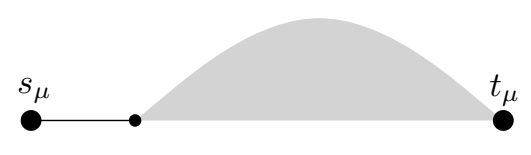

Figure 15: An example of an $S$-node with a tail at $s_{\mu}$.

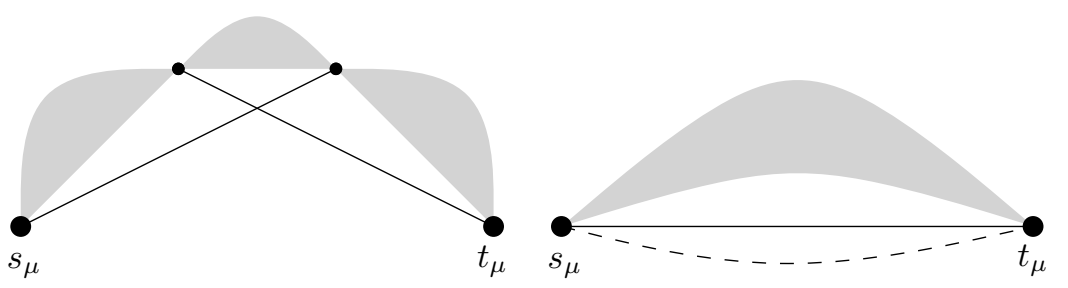

(a)

(b) Case (i)

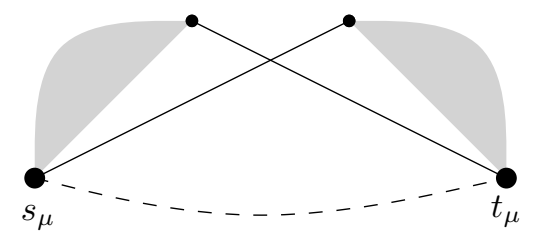

(c) Case (ii)

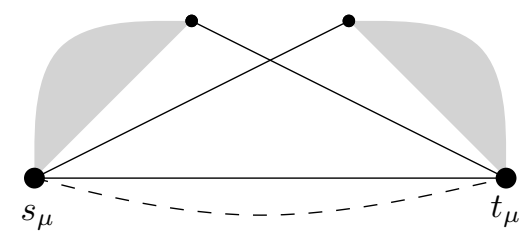

(d) Case (iii)

Figure 16: Illustration of Properties 3 and 4 (a) The pertinent graph of an $R$-node $\mu$. (b)-(d)The pertinent graph of a $P$-node $\mu$ whose reference edges (dashed) is not crossed in $G_{\mu}^{+}$.

Property 4 Let $\mu$ be a P-node of $T$ such that the reference edge $\left(s_{\mu}, t_{\mu}\right)$ is not crossed in $G_{\mu}^{+}$and belongs to the outer face of $G_{\mu}^{+}$, then one of the following cases holds:

(i) $\mu$ has two children one of which is a $Q$-node and the other one is either an $R$-node or an $S$-node.

(ii) $\mu$ has two children and none of them is a $Q$-node. Both are $S$-nodes, one of them has a tail at $s_{\mu}$, and the other one has a tail at $t_{\mu}$. Also, the two edges associated with these two tails cross each other in $G$.

(iii) $\mu$ has three children and one of them is a $Q$-node. For the remaining two children case (ii) applies.

Thus, if $\mu$ is a $P$-node of $T$ such that the edge $\left(s_{\mu}, t_{\mu}\right)$ is not crossed and belongs 


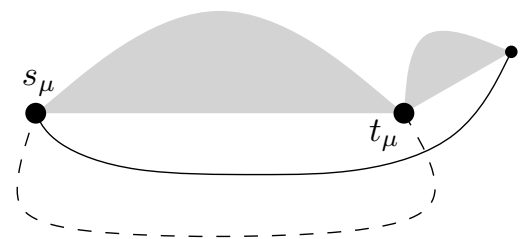

(a) Case $k=2$

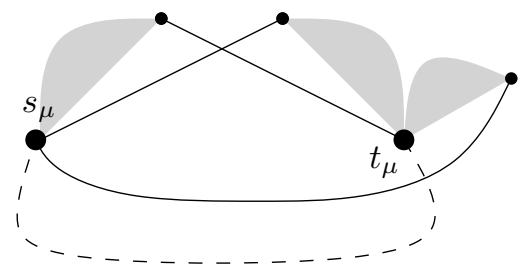

(c) Case $k=3$ (ii)

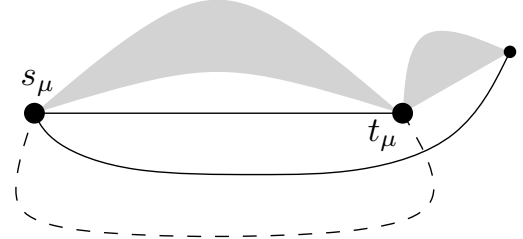

(b) Case $k=3$ (i)

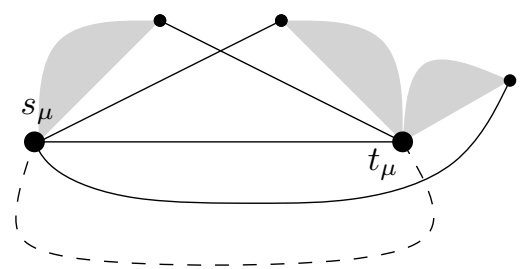

(d) Case $k=4$

Figure 17: Illustration of Property 5 The pertinent graph of a $P$-node $\mu$ whose reference edges (dashed) is crossed in $G_{\mu}^{+}$.

to the outer face of $G_{\mu}^{+}$then it can have at most three children as described in Property 4. On the other hand, if the reference edge $\left(s_{\mu}, t_{\mu}\right)$ is crossed in $G_{\mu}^{+}$, then $\mu$ can have up to four children and the following property applies [14, see also Figures $17(\mathrm{a})[17(\mathrm{~d})$ for an illustration.

Property 5 Let $\mu$ be a P-node of $T$ such that the reference edge $\left(s_{\mu}, t_{\mu}\right)$ is crossed in $G_{\mu}^{+}$. Then $\mu$ has $2 \leq k \leq 4$ children and one of them is an $S$-node with a tail at $s_{\mu}$ or at $t_{\mu}$. If $k=2$, the other child is either a $Q-/ S-/$ or an $R$-node. If $k=3$, then for the remaining children either case (i) or case (ii) of Property 4 holds. If $k=4$, then for the remaining children Property 4 (iii) holds.

If $\mu$ is a $P$-node of $T$ such that the edge $\left(s_{\mu}, t_{\mu}\right)$ is crossed in $G_{\mu}^{+}$, as described in Property 5 , then it has an $S$-node with a tail at $s_{\mu}$ or at $t_{\mu}$ as a child. In the first case, we call $\mu$ a $P$-node with a tail at $s_{\mu}$, in the second case we call $\mu$ a $P$-node with a tail at $t_{\mu}$. Moreover, since the graph is outer 1-plane, the edge of $G_{\mu}$ associated with the tail at $s_{\mu}\left(t_{\mu}\right)$ crosses another edge, represented by a $Q$-node $\psi$ in $T$, having $t_{\mu}\left(s_{\mu}\right)$ as an end vertex. This implies that in fact, $\mu$ and $\psi$ are two children of an $S$-node $\nu$ in $T$ [14] (see also Figure 18). This observation will be used later and in the next property, that is derived from Lemma 7 in [14.

Property 6 Let $\mu$ be an $S$-node of $T$. Let $\eta_{1}, \eta_{2}, \ldots, \eta_{k}$ be the $k$ children of $\mu$ in $T$, such that $t_{\eta_{i-1}}=s_{\eta_{i}}$, for $i=2, \ldots, k$. Then for each $1 \leq i \leq k$, one of the following cases applies: 


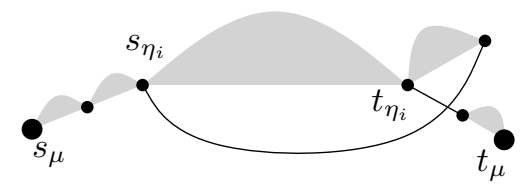

Figure 18: Illustration of Property 6 The pertinent graph of an $S$-node $\mu$ with a child $\eta_{i}$ that is a $P$-node with a tail at $s_{\eta_{i}}$.

(i) $\eta_{i}$ is either a $Q$-node, an $R$-node or a $P$-node such that the reference edge $\left(s_{\eta_{i}}, t_{\eta_{i}}\right)$ is not crossed in $G_{\eta_{i}}^{+}$.

(ii) $\eta_{i}$ is a $P$-node with a tail at $s_{\eta_{i}}$ and $\eta_{i+1}(i<k)$ is a $Q$-node.

(iii) $\eta_{i}$ is a $P$-node with a tail at $t_{\eta_{i}}$ and $\eta_{i-1}(i>1)$ is a $Q$-node.

A Universal Set of Slopes. We define a universal set of slopes used by algorithm BO1P-DRAWER to compute an outer 1-planar drawing of every biconnected outer 1-planar graph $G$ with maximum degree $\Delta$. Let $\alpha=\frac{\pi}{2 \Delta}$ and observe that $0<\alpha \leq \frac{\pi}{6}$ when $\Delta \geq 3$. Denote by blue slopes the set of slopes defined as $b_{i}=(i-1) \alpha$, for $i=1,2, \ldots, 2 \Delta$. For each of the $2 \Delta$ blue slopes, we also define two red slopes as $r_{i}^{-}=b_{i}-\varepsilon$ and $r_{i}^{+}=b_{i}+\varepsilon$, for $i=1,2, \ldots, 2 \Delta$. The value of $\varepsilon$ can be any number such that:

$$
0<\varepsilon<\hat{\varepsilon}=\alpha-\arctan \left(\frac{\tan (\alpha)}{1+2 \tan (3 / 2 \alpha) \tan (\alpha / 2)-2 \tan (\alpha) \tan (\alpha / 2)}\right)
$$

The reason of this choice will be clarified in the proof of Lemma 10 . We now show that $\hat{\varepsilon}$ is a positive value that depends only on $\Delta$. The tangent function is monotonically increasing in the range $\left(-\frac{\pi}{2}, \frac{\pi}{2}\right)$, thus $\tan (3 / 2 \alpha)>\tan (\alpha)$ $\left(0<2 \alpha \leq \frac{\pi}{3}\right)$. This implies that the denominator of the argument of the arctangent function is strictly larger than 1, and the overall argument is strictly less than $\tan (\alpha)$. Since the arctangent function is monotonically increasing, the term subtracted from $\alpha$ on the right-hand side of the equation is strictly smaller than $\alpha$ and therefore $\hat{\varepsilon}$ is greater than zero. The union of the blue and red slopes defines the universal set of slopes $\mathcal{S}_{\Delta}$ of size $6 \Delta$.

Algorithm Overview. Algorithm BO1P-DRAWER works in a similar way as BP-DrAWER. It takes as input a biconnected outer 1-planar graph $G$ with maximum degree $\Delta$ and returns an outer 1-planar straight-line drawing $\Gamma$ of $G$ that uses only slopes in $\mathcal{S}_{\Delta}$. Figure 19 shows a drawing computed by algorithm BO1P-DrAWER. The algorithm first constructs an outer 1-planar embedding of $G$, together with the $S P Q R$-tree $T$ of $G$ rooted at a $Q$-node $\rho$, whose (only) child is denoted as $\xi$. Then BO1P-Drawer draws $G$ by visiting $T$ bottom-up, 
handling $\rho$ and $\xi$ together as a special case. At each step a node $\mu$ of $T$ different from $\xi$ is processed and a drawing $\Gamma_{\mu}$ of its pertinent graph $G_{\mu}$ is computed. If $\mu$ is a $Q$-node, then its pertinent graph is an edge $\left(s_{\mu}, t_{\mu}\right)$ and is drawn as a horizontal segment of unit length. If $\mu$ is not a $Q$-node (i.e., is not a leaf), $\Gamma_{\mu}$ is computed by properly combining the already computed drawings of the pertinent graphs of the children of $\mu$. Let $s_{\mu}$ and $t_{\mu}$ be the poles of $\mu$. Then for each drawing $\Gamma_{\mu}$ the algorithm maintains the following three invariants.

I1. $\Gamma_{\mu}$ is outer 1-planar.

I2. $\Gamma_{\mu}$ uses only slopes in $\mathcal{S}_{\Delta}$.

I3. $\Gamma_{\mu}$ is contained in a triangle $\tau_{\mu}$ such that $s_{\mu}$ and $t_{\mu}$ are placed at the corners of its base. Also, $\beta_{\mu}<\left(\Delta\left(s_{\mu}\right)+1 / 2\right) \alpha$ and $\gamma_{\mu}<\left(\Delta\left(t_{\mu}\right)+1 / 2\right) \alpha$, where $\beta_{\mu}$ and $\gamma_{\mu}$ are the internal angles of $\tau_{\mu}$ at $s_{\mu}$ and $t_{\mu}$, respectively.

Observe that Invariant I3 is well defined. In fact, for a node $\mu$ of $T$ different from $\xi$, we have that $\Delta\left(s_{\mu}\right) \leq \Delta-1$ and $\Delta\left(t_{\mu}\right) \leq \Delta-1$, which implies $\beta_{\mu}<$ $(\Delta-1 / 2) \alpha=\pi / 2-\alpha / 2$ and $\gamma_{\mu}<(\Delta-1 / 2)=\pi / 2-\alpha / 2$.

The root $\rho$ of $T$ and its unique child $\xi$ are handled in a special way. The drawing $\Gamma_{\mu}$ of $G_{\mu}$ is computed by combining the drawings $\Gamma_{\eta_{1}}, \Gamma_{\eta_{2}}, \ldots, \Gamma_{\eta_{k}}$ of the pertinent graphs $G_{\eta_{1}}, G_{\eta_{2}}, \ldots, G_{\eta_{k}}$ of the children $\eta_{1}, \eta_{2}, \ldots, \eta_{k}$ of $\mu$. To this aim, if necessary, the drawings $\Gamma_{\eta_{1}}, \Gamma_{\eta_{2}}, \ldots, \Gamma_{\eta_{k}}$ are manipulated similarly as described for algorithm BP-DrAWER. More specifically, each drawing $\Gamma_{\eta_{j}}$ $(1 \leq j \leq k)$ can be arbitrarily scaled, or it can be rotated by an angle $c \cdot \alpha$, with $c$ integer. Note that invariant $\mathbf{I 2}$ is maintained if a drawing is rotated by an angle $c \cdot \alpha$. In fact, each blue slope $b_{i}$, for $i=1,2, \ldots, 2 \Delta$, used in $\tau_{\eta_{j}}$ will be transformed into another blue slope $b_{i+c}=b_{i}+c \cdot \alpha=(i-1+c) \alpha$, where $i+c$ is considered modulo $2 \Delta$. Similarly, any red slope will be transformed into another red slope. Similarly, due to the symmetric choice of the slopes, a horizontal flip of the drawing does not affect invariant I2. Also, two drawings $\Gamma_{\eta_{i}}$ and $\Gamma_{\eta_{j}}(1 \leq i<j \leq k)$ that share either two poles or one pole can be attached.

Before describing how BO1P-DrAWER works in more details, we need to distinguish between $P$-nodes for which Property 4 holds, and $P$-nodes for which Property 5 holds instead. Recall that Property 4 applies if the reference edge of the node is not crossed in the augmented pertinent graph, whereas Property 5 applies if the reference edge is crossed. Let $\varphi$ be a $P$-node for which Property 5 applies, then $\varphi$ has a tail at $s_{\varphi}$ or at $t_{\varphi}$. We also know that $\varphi$ is one of the

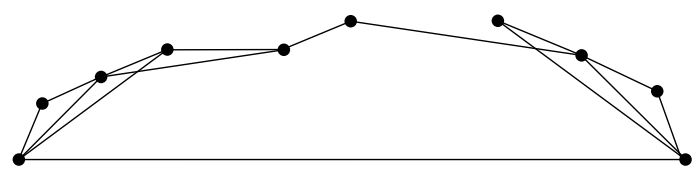

Figure 19: An outer 1-planar drawing computed by algorithm BO1P-Drawer. The input graph is the same as shown in Figure 5 

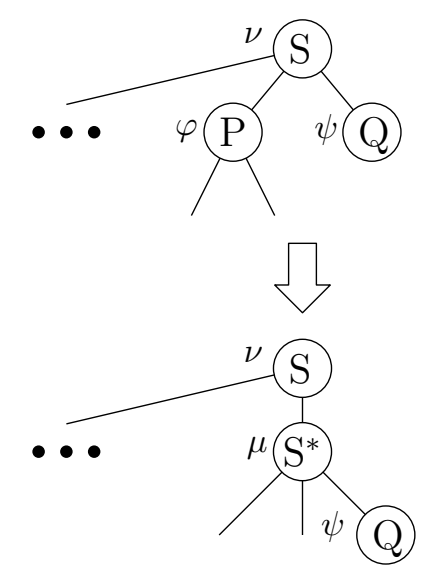

(a)
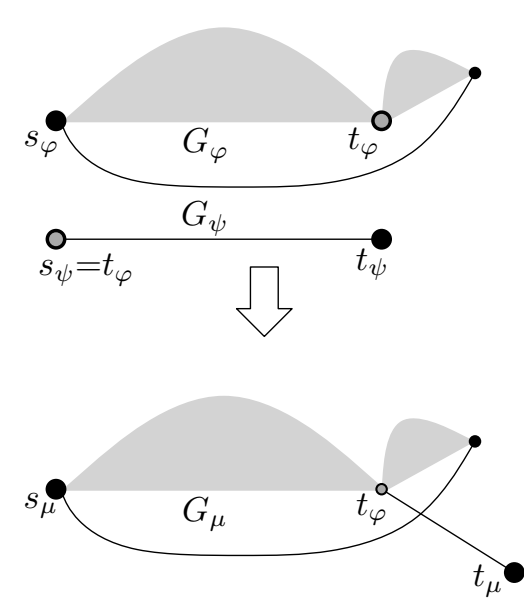

(b)

Figure 20: Illustration of an $S^{*}$-node: (a) Transformation of the $S P Q R$-tree; (b) Merging $G_{\mu}$ and $G_{\eta}$ into $G_{\phi}$.

children of an $S$-node, say $\nu$, and it shares a pole with a $Q$-node, denoted as $\psi$ (also a child of $\nu$ ). We replace $\varphi$ with a new node $\mu$, that, for the sake of description, is called an $S^{*}$-node and we make $\psi$ a child of $\mu$. Also, the children of $\varphi$ become children of $\mu$. If $\varphi$ and $\psi$ were the only two children of $\nu$, then we also replace $\nu$ with $\mu$. The pertinent graph of $\mu$ is $G_{\mu}=G_{\varphi} \cup G_{\psi}$, while the reference edge of $\mu$ is $\left(s_{\varphi}, t_{\psi}\right)$, if $\varphi$ has a tail at $s_{\varphi}$, or $\left(s_{\psi}, t_{\varphi}\right)$, if $\varphi$ has a tail at $t_{\mu}$. See also Figure 20. By means of this transformation we can consider only $P$-nodes that have their reference edge uncrossed in their augmented pertinent graphs, i.e., for which Property 4 holds. Similarly we can handle just $S$-nodes whose children are $S^{*}$ - $/ R$-nodes or $P$-nodes for which Property 4 holds.

Embedding and $S P Q R$-tree Computation. Algorithm BO1P-DraWER first computes an outer 1-planar embedding of $G$. Then, algorithm BO1PDRAWER computes the $S P Q R$-tree $T$ of $G$, and roots it at a $Q$-node $\rho$ (whose only child is denoted as $\xi$ ) such that the edge associated with it is not crossed and belongs to the boundary of the outer face of $G$. Finally, algorithm BO1PDraWER creates the $S^{*}$-nodes, if necessary. We now show that the described choice of the root is always possible and then give a property that describes the implication of this choice.

Lemma 7 There exists an edge e of $G$, such that e is not crossed and it belongs to the outer face of $G$.

Proof: Suppose by contradiction that all the edges of the outer face of $G$ are crossed, i.e., the outer face of $G$ is composed of a set of edge fragments such that 
none of them is trivial. Then, replace each crossing in $G$ with a dummy vertex, and denote the planarized resulting graph by $G^{*}$. Each dummy vertex has degree four and all its four neighbors are real vertices placed on the boundary of the outer face. It follows that, walking around the boundary of the outer face of $G^{*}$, there are no two consecutive dummy vertices. Also, there are no two consecutive real vertices, as otherwise the edge fragment connecting these two real vertices would be a trivial fragment that corresponds to an uncrossed edge. Thus, walking around the boundary of the outer face of $G^{*}$ we find an alternating sequence of real and dummy vertices. Consider now the subgraph induced by the vertices (both real and dummy) that belong to the boundary of the outer face of $G^{*}$, minus the chords (i.e., edges that do not belong to the outer face) between real vertices. Denote this subgraph of $G^{*}$ as $G^{\prime}$, and observe it is outerplanar, since it is a subgraph of a planar graph and all its vertices are on the outer face. In what follows we distinguish two cases depending on whether $G^{\prime}$ is biconnected or not.

Suppose first that $G^{\prime}$ is biconnected. Denote by $n^{\prime}$ and $m^{\prime}$ the number of vertices and the number of edges of $G^{\prime}$, respectively. Note that $n^{\prime}$ is an even integer because real and dummy vertices alternate along the boundary of the outer face. We now count the number of edges of $G^{\prime}$. Since each edge in $G^{\prime}$ has exactly one dummy end-vertex, then $m^{\prime}$ is equal to the sum of the degree of the dummy vertices. Since we have $\frac{n^{\prime}}{2}$ dummy vertices in $G^{\prime}$, each having degree 4 , we have $m^{\prime}=4 \cdot \frac{n^{\prime}}{2}=2 n^{\prime}$. Denote by $\mathcal{F}^{\prime}$ the set of faces of $G^{\prime}$. By Euler's formula we have that $\left|\mathcal{F}^{\prime}\right|=m^{\prime}-n^{\prime}+2=n^{\prime}+2$. Also, denote by $\operatorname{deg}(f)$, the degree of a face $f \in \mathcal{F}^{\prime}$, i.e., the number of edges that belong to face $f$. The outer face has degree $n^{\prime}$, while all the internal faces have degree at least 4 . Indeed, a triangular face would imply a chord between two real vertices (which would correspond to an uncrossed edge on the outer face of $G$ ) or between two dummy vertices (which does not exist by construction). Thus, we have that $2 m^{\prime}=\sum_{f \in \mathcal{F}^{\prime}} \operatorname{deg}(f) \geq n^{\prime}+4\left(n^{\prime}+2-1\right)$, which implies that $2 m^{\prime} \geq 5 n^{\prime}+4$. Since $m^{\prime}=2 n^{\prime}$, we obtain $4 n^{\prime} \geq 5 n^{\prime}+4$, i.e., $n^{\prime} \leq-4$, contradicting $n^{\prime}>0$.

Suppose now that $G^{\prime}$ contains one or more cut vertices. Since $G$ is biconnected, every cut vertex of $G^{\prime}$ is a dummy vertex and it is shared by exactly two components (because every dummy vertex has degree four). Also, there exists at least one component $C$ in $G^{\prime}$ containing exactly one cut vertex. Similar to the biconnected case, denote by $n_{C}^{\prime}$ and $m_{C}^{\prime}$ the number of vertices and the number of edges of $C$, respectively. Also in this case, $n_{C}^{\prime}$ is an even integer and $m_{C}^{\prime}$ is equal to the sum of the degree in $C$ of the dummy vertices. The number of dummy vertices in $C$ is $\frac{n_{C}^{\prime}}{2}$, each having degree 4 except for the cut vertex (that has degree 2 ). Thus $m_{C}^{\prime}=4 \cdot\left(\frac{n_{C}^{\prime}}{2}-1\right)+2=2 n_{C}^{\prime}-2$. Denote by $\mathcal{F}_{C}^{\prime}$ the set of faces of $C^{\prime}$. By Euler's formula we have that $\left|\mathcal{F}_{C}^{\prime}\right|=m_{C}^{\prime}-n_{C}^{\prime}+2=n_{C}^{\prime}$. The outer face has degree $n_{C}^{\prime}$, while all the internal faces have degree at least 4. Thus, we have that $2 m_{C}^{\prime}=\sum_{f \in \mathcal{F}_{C}^{\prime}} \operatorname{deg}(f) \geq n_{C}^{\prime}+4\left(n_{C}^{\prime}-1\right)$, which implies that $2 m_{C}^{\prime} \geq 5 n_{C}^{\prime}-4$. Since $m_{C}^{\prime}=2 n_{C}^{\prime}-2$, we obtain $4 n_{C}^{\prime}-4 \geq 5 n_{C}^{\prime}-4$, i.e., $n_{C}^{\prime} \leq 0$, contradicting $n_{C}^{\prime}>0$. 
Choosing the root of $T$ as a non-crossed edge on the outer face, implies the following property.

Property 7 If $T$ is rooted at a $Q$-node associated with an edge e that is on the outer face and is not crossed, then for every $P$-node $\mu$ of $T$ either the reference edge of $\mu$ is crossed or it belongs to the outer face of $G_{\mu}^{+}$.

Proof: We show that if the reference edge of $\mu$ is not crossed, it belongs to the outer face of $G_{\mu}^{+}$. If $\mu$ coincides with the child $\xi$ of the root, then this is trivially true by the choice of the root. Consider a $P$-node $\mu$ that is not the child of the root and whose reference edge $\left(s_{\mu}, t_{\mu}\right)$ is not crossed. The reference edge represents a subgraph of $G$ containing at least one vertex distinct from $s_{\mu}$ and $t_{\mu}$ (otherwise the subgraph would correspond to a $Q$-node in $T$ and it would be the root of $T$ ); all the vertices of this subgraph are on the outer face of $G$ in the given outer 1-planar embedding. It follows that $\left(s_{\mu}, t_{\mu}\right)$ must be on the external face of $G_{\mu}^{+}$.

A consequence of Property 7 is that for every $P$-node of $T$ either Property 4 or Property 5 holds. Also, if the $P$-node is the child of the root $\xi$, only case (ii) of Property 4 holds (otherwise there would be a multiple edge between the poles of $\xi$ ).

The Drawing Algorithm. In the next lemmas we show how BO1P-Drawer computes a drawing $\Gamma_{\mu}$ of the pertinent graph $G_{\mu}$ of a node $\mu$, depending on the type of $\mu$. Recall that $\xi$ is the (only) child of the root $\rho$ of $T$ and that the leaves of $T$ are $Q$-nodes by definition.

Lemma 8 Let $\mu$ be an $S$-node different from $\xi$. Then $G_{\mu}$ admits a straight-line drawing $\Gamma_{\mu}$ that respects Invariants I1, I2, and $\mathbf{I}$.

Proof: Let $\eta_{1}, \eta_{2}, \ldots, \eta_{k}$ be the $k \geq 2$ children of $\mu$ in $T$. In order to construct $\Gamma_{\mu}$, the drawings $\Gamma_{\eta_{1}}, \Gamma_{\eta_{2}}, \ldots, \Gamma_{\eta_{k}}$ of the pertinent graphs of $\eta_{1}, \eta_{2}, \ldots, \eta_{k}$ are combined as follows, see also Figure 7. If $k>2$, then, in order to satisfy Invariant I2, we need that the height of $\tau_{\eta_{i}}$ is less than the minimum between the height of $\tau_{\eta_{1}}$ and the height of $\tau_{\eta_{k}}$. To this aim, BO1P-Drawer scales down $\Gamma_{\eta_{i}}$, for $i=2, \ldots, k-1$, if necessary. Then, $\Gamma_{\eta_{1}}, \Gamma_{\eta_{2}}, \ldots, \Gamma_{\eta_{k}}$ are attached to each other so that the bases of the triangles $\tau_{\eta_{1}}, \tau_{\eta_{2}}, \ldots, \tau_{\eta_{k}}$ are all contained in the same horizontal straight line, and such that all the vertices of $G_{\mu}$ are above the horizontal segment $\overline{s_{\mu} t_{\mu}}$. Invariant $\mathbf{I} 1$ holds by construction because we combined the drawings in such a way that they do not intersect each other (except at common vertices). Invariant $\mathbf{I} 2$ holds since the slopes of $\Gamma_{\eta_{i}}$, for $i=1, \ldots, k$, have not been changed. Invariant $\mathbf{I} 3$ holds because it holds for $\Gamma_{\eta_{1}}$ and $\Gamma_{\eta_{k}}$ and all triangles $\tau_{\eta_{i}}$ (for $i=1, \ldots, k$ ) have a height smaller than that of $\tau_{\eta_{1}}$ and $\tau_{\eta_{k}}$ (due to the scaling).

In order to prove the next lemma, we observe that the operation bend $\left(\Gamma_{\mu}, \beta_{\mu}^{*}\right.$, $\gamma_{\mu}^{*}$ ), which is defined in Section 3 , can be applied also to the drawings of $S$-nodes computed as shown in the proof of Lemma 8 . 


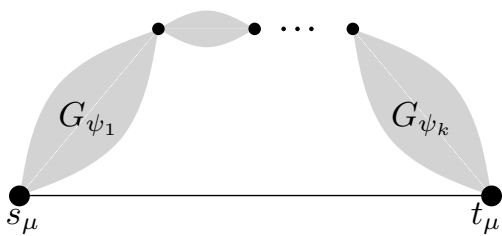

(a)

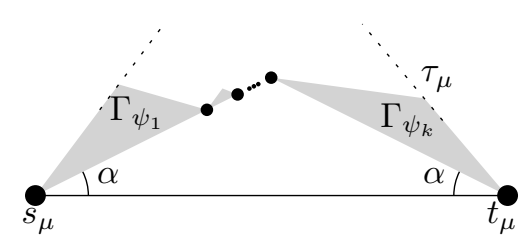

(b)

Figure 21: Illustration of Lemma 9. (a) The pertinent graph $G_{\mu}$ of an $P$-node $\mu$ with two children that are a $Q$-node and an $S$-node. (b) The outer 1-planar drawing of $G_{\mu}$.

Lemma 9 Let $\mu$ be a P-node different from $\xi$. Then $G_{\mu}$ admits a straight-line drawing $\Gamma_{\mu}$ that respects Invariants $\mathbf{I} \mathbf{1}, \mathbf{I} 2$, and $\mathbf{I}$ 3.

Proof: Recall that, thanks to the definition of the $S^{*}$-nodes, here we need to handle only $P$-nodes whose reference edges are not crossed in their augmented pertinent graphs. By Property 4 one of the following cases applies: $(i) \mu$ has two children one of which is a $Q$-node and the other one is either an $R$-node or an $S$-node. (ii) $\mu$ has two children and none of them is a $Q$-node. Then both are $S$-nodes, one of them has a tail at $s_{\mu}$ and the other one has a tail at $t_{\mu}$. Also, the two edges associated with these two tails cross each other in $G$. (iii) $\mu$ has three children and one of them is a $Q$-node. For the remaining two children case $(i i)$ applies.

If we are in case $(i)$, denote by $\eta$ the child of $\mu$ which is not a $Q$-node. Suppose first that $\eta$ is an $S^{*}$-node or an $R$-node. As it will be shown in the proofs of Lemmas 10 and 11. in these cases the horizontal blue slope $b_{1}$ is not used in $\Gamma_{\eta}$. Thus, the edge $\left(s_{\mu}, t_{\mu}\right)$ can be safely drawn using $b_{1}$ without modifying $\Gamma_{\eta}$, and all invariants hold. Suppose now that $\eta$ is an $S$-node and see also Figure 21. We apply the following operation $\operatorname{bend}\left(\Gamma_{\eta}, \alpha, \alpha\right)$. Then we draw the edge $\left(s_{\mu}, t_{\mu}\right)$ using the horizontal blue slope $b_{1}$. Invariants $\mathbf{I} 1$ and I2 hold by construction. Also, $\Gamma_{\mu}$ is contained in a triangle $\tau_{\mu}$ such that $s_{\mu}$ and $t_{\mu}$ are placed at the corners of its base. Moreover, we have that $\Delta\left(s_{\mu}\right)=$ $\Delta\left(s_{\eta}\right)+1=\Delta\left(s_{\psi_{1}}\right)+1$, where $\psi_{1}$ is the child of $\eta$ such that $s_{\eta}=s_{\psi_{1}}$. Also, $\beta_{\mu}=\beta_{\psi_{1}}+\alpha<\left(\Delta\left(s_{\psi_{1}}\right)+1 / 2\right) \alpha+\alpha=\left(\Delta\left(s_{\psi_{1}}\right)+3 / 2\right) \alpha=\left(\Delta\left(s_{\mu}\right)+1 / 2\right) \alpha$. Similarly, $\Delta\left(t_{\mu}\right)=\Delta\left(t_{\eta}\right)+1=\Delta\left(t_{\psi_{k}}\right)+1$, where $\psi_{k}$ is the child of $\eta$ such that $t_{\eta}=t_{\psi_{k}}$. Also, $\gamma_{\mu}=\gamma_{\psi_{k}}+\alpha<\left(\Delta\left(t_{\psi_{k}}\right)+1 / 2\right) \alpha+\alpha=\left(\Delta\left(t_{\psi_{k}}\right)+3 / 2\right) \alpha=$ $\left(\Delta\left(t_{\mu}\right)+1 / 2\right) \alpha$. Hence, Invariant $\mathbf{I 3}$ holds.

If we are in case $(i i)$, denote by $\eta_{1}$ the child of $\mu$ that is an $S$-node with a tail at $t_{\mu}$, and as $\eta_{2}$ the child of $\mu$ that is an $S$-node with a tail at $s_{\mu}$. See Figure 22 for an illustration. Recall that $s_{\eta_{1}}=s_{\eta_{2}}=s_{\mu}$ and $t_{\eta_{1}}=t_{\eta_{2}}=t_{\mu}$. We modify the drawing $\Gamma_{\eta_{1}}$ as follows. We first rotate $\Gamma_{\eta_{1}}$ so that the segment $\overline{s_{\eta_{1}} t_{\eta_{1}}}$ uses the blue slope $b_{2}$. Then we redraw the tail of $\eta_{1}$ using the red slope $r_{2 \Delta}^{+}=b_{2 \Delta}+\varepsilon$ and so that $s_{\eta_{1}}$ and $t_{\eta_{1}}$ are horizontally aligned. Similarly, we modify the drawing $\Gamma_{\eta_{2}}$. We rotate $\Gamma_{\eta_{2}}$ so that the segment $\overline{s_{\eta_{2}} t_{\eta_{2}}}$ uses the blue 


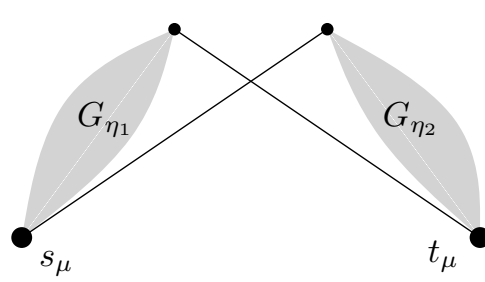

(a)

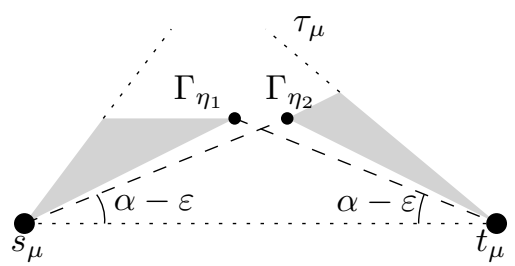

(b)

Figure 22: Illustration of Lemma 9. (a) The pertinent graph $G_{\mu}$ of an $P$-node $\mu$ with two children that are $S$-nodes. (b) The outer 1-planar drawing of $G_{\mu}$.

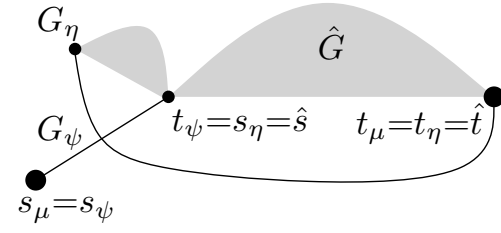

(a)

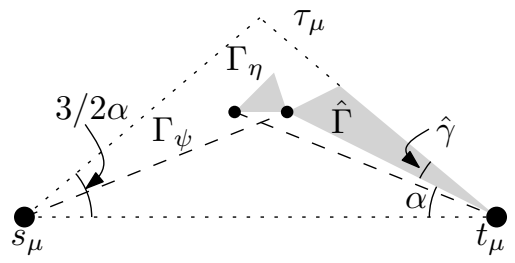

(b)

Figure 23: Illustration of Lemma 10 (a) The pertinent graph $G_{\mu}$ of an $S^{*}$-node $\mu$. (b) The outer 1-planar drawing of $G_{\mu}$.

slope $b_{2 \Delta}$ and redraw the tail of $\eta_{2}$ using the red slope $r_{2}^{-}=b_{2}-\varepsilon$ and so that $s_{\eta_{2}}$ and $t_{\eta_{2}}$ are horizontally aligned. Finally, we attach $\Gamma_{\eta_{1}}$ and $\Gamma_{\eta_{2}}$ (scaling one of them if necessary). Invariants $\mathbf{I} \mathbf{1}$ and $\mathbf{I} 2$ hold by construction. Also, $\Gamma_{\mu}$ is contained in a triangle $\tau_{\mu}$ such that $s_{\mu}$ and $t_{\mu}$ are placed at the corners of its base. Moreover, we have that $\Delta\left(s_{\mu}\right)=\Delta\left(s_{\eta_{1}}\right)+1$, and $\beta_{\mu}=\beta_{\eta_{1}}+\alpha<\left(\Delta\left(s_{\eta_{1}}\right)+\right.$ $1 / 2) \alpha+\alpha=\left(\Delta\left(s_{\eta_{1}}\right)+3 / 2\right) \alpha=\left(\Delta\left(s_{\mu}\right)+1 / 2\right) \alpha$. Similarly, $\Delta\left(t_{\mu}\right)=\Delta\left(t_{\eta_{2}}\right)+1$, and $\gamma_{\mu}=\gamma_{\eta_{2}}+\alpha<\left(\Delta\left(t_{\eta_{2}}\right)+1 / 2\right) \alpha+\alpha=\left(\Delta\left(t_{\eta_{2}}\right)+3 / 2\right) \alpha=\left(\Delta\left(t_{\mu}\right)+1\right) \alpha$. Hence, Invariant $\mathbf{I} 3$ holds.

If we are in case $(i i i)$, we can use the same construction as in case $(i i)$. Note that the edge $\left(s_{\mu}, t_{\mu}\right)$ can be safely drawn using the horizontal blue slope $b_{1}$. All invariants hold also in this case.

Lemma 10 Let $\mu$ be an $S^{*}$-node different from $\xi$. Then $G_{\mu}$ admits a straightline drawing $\Gamma_{\mu}$ that respects Invariants I1, I2, and $\mathbf{I}$.

Proof: Denote by $\eta$ the child of $\mu$ that is an $S$-node with a tail at either $s_{\mu}$ or $t_{\mu}$. Suppose that $\eta$ has a tail at $t_{\mu}$ (the case when the tail is at $s_{\mu}$ is symmetric) and see Figure 23 for an illustration. Denote by $\psi$ the child of $\mu$ that is a $Q$-node having $t_{\psi}=s_{\eta}$ and $s_{\psi}=s_{\mu}$ as poles. Finally denote by $\eta_{1}, \eta_{2}, \ldots, \eta_{k}$ the remaining children of $\mu$. Recall that $s_{\eta_{1}}=\cdots=s_{\eta_{k}}=s_{\eta}$ 


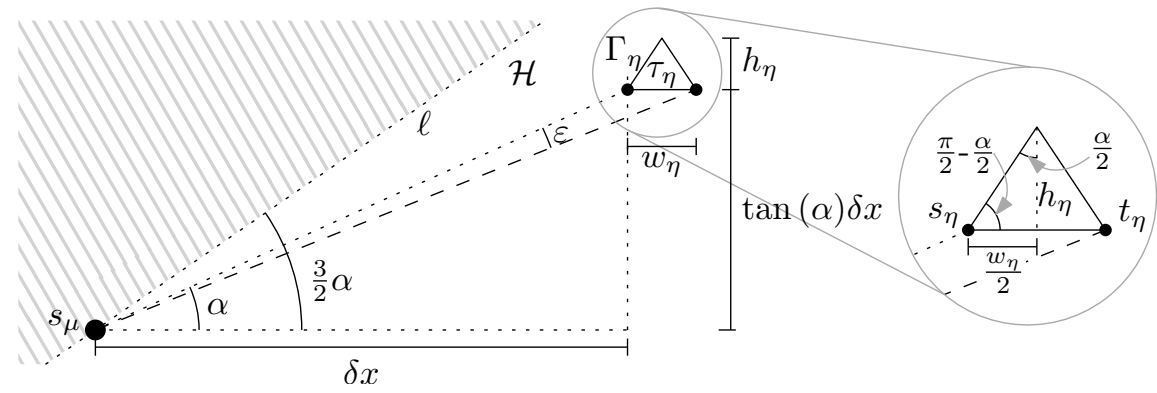

Figure 24: Illustration for the proof of Invariant I3 for $S^{*}$-nodes (Lemma 10 .

and that $t_{\eta_{1}}=\cdots=t_{\eta_{k}}=t_{\eta}$. Denote by $\hat{G}$ the subgraph of $G_{\mu}$ obtained by the parallel composition of $\eta_{1}, \eta_{2}, \ldots, \eta_{k}$, and denote by $\hat{s}$ and $\hat{t}$ its poles, coinciding with $s_{\eta_{1}}=\cdots=s_{\eta_{k}}=s_{\eta}$ and $t_{\eta_{1}}=\cdots=t_{\eta_{k}}=t_{\eta}$, respectively. We first compute a drawing $\hat{\Gamma}$ of $\hat{G}$; if $k=1, \hat{\Gamma}$ coincides with $\Gamma_{\eta_{1}}$, otherwise it is obtained by combining the drawings $\Gamma_{\eta_{1}}, \Gamma_{\eta_{2}}, \ldots, \Gamma_{\eta_{k}}$ with the same technique described for $P$-nodes in the proof of Lemma 9 (recall that indeed they were children of a $P$-node before the creation of the $S^{*}$-node). In both cases we rotate $\hat{\Gamma}$ so that the base of its bounding triangle uses the blue slope $b_{2 \Delta}$. Then we attach $\Gamma_{\eta}$ to $\hat{\Gamma}$ (after $\Gamma_{\eta}$ has been horizontally flipped). Also, we scale $\Gamma_{\eta}$ so that its tail can be redrawn by using the red slope $r_{2 \Delta}^{+}$and such that $t_{\eta}=t_{\mu}$ coincides with $\hat{t}$. Finally, we redraw the edge associated with $\psi$, starting from the point representing $t_{\psi}=s_{\eta}$, using the red slope $r_{2}^{-}$and stretching it enough that $s_{\psi}=s_{\mu}$ and $t_{\mu}$ are horizontally aligned. See also Figure 23(b) for an illustration. Invariants $\mathbf{I} \mathbf{1}$ and $\mathbf{I} \mathbf{2}$ hold by construction. Consider now Invariant I3; by construction $\Gamma_{\mu}$ is contained in a triangle $\tau_{\mu}$ such that $s_{\mu}$ and $t_{\mu}$ are placed at the corners of its base. The drawing $\hat{\Gamma}$ satisfies Invariant $\mathbf{I} 3$ either by Lemma 9 (if $k>1$ ) or because it coincides with $\Gamma_{\eta_{1}}$. More precisely, $\hat{\Gamma}$ is contained in a triangle $\hat{\tau}$ such that $\hat{s}$ and $\hat{t}$ are the corner of its base, with $\hat{\beta}<(\Delta(\hat{s})+1 / 2) \alpha$ and $\hat{\gamma}<(\Delta(\hat{t})+1 / 2) \alpha$ (with obvious meaning of the symbols). The angle $\gamma_{\mu}$ at $t_{\mu}$ is equal to $\hat{\gamma}+\alpha$ and therefore $\gamma_{\mu}<(\Delta(\hat{t})+1 / 2) \alpha+\alpha$. Since $\Delta\left(t_{\mu}\right)=\Delta(\hat{t})+1$, we obtain, $\gamma_{\mu}<\left(\Delta\left(t_{\mu}\right)+1 / 2\right) \alpha$.

We now look at $\beta_{\mu}$. For the sake of description, we denote by $\Gamma_{\eta}$ the drawing of $G_{\eta}$ minus the tail of $\eta$ (i.e., minus an edge), and as $\tau_{\eta}$ the surrounding triangle of $\Gamma_{\eta}$. We now prove that the line $\ell$ passing through $s_{\mu}$ with slope $3 / 2 \alpha$ does not cross the drawing of $\Gamma_{\eta}$, i.e., $\Gamma_{\eta}$ is placed in the half-plane $\mathcal{H}$ defined by $\ell$ and containing the segment $\overline{s_{\mu} t_{\mu}}$. See Figure 24 for an illustration. Denote by $\delta x$ the horizontal distance between the point where $s_{\mu}$ is drawn and the leftmost corner of $\tau_{\eta}$. Also, denote as $h_{\eta}$ the height of $\tau_{\eta}$. To prove that our condition is satisfied it is sufficient to show that:

$$
\tan (3 / 2 \alpha) \delta x>\tan (\alpha) \delta x+h_{\eta}
$$

Let $w_{\eta}$ be the length of the base of $\tau_{\eta}$. Then the worst case, i.e., the case when $h_{\eta}$ is maximized, is realized if the degree of $s_{\eta}$ and $t_{\eta}$ is $\Delta-1$ (it cannot be $\Delta$ 
because of the edges $\left(s_{\eta}, t_{\mu}\right)$ and $\left.\left(s_{\mu}, t_{\eta}\right)\right)$. In such a case we have that the angle of $\tau_{\eta}$ at $s_{\eta}$ (at $\left.t_{\eta}\right)$ is strictly less than $(\Delta-1+1 / 2) \alpha=\pi / 2-\alpha / 2$. We have

$$
h_{\eta}<\frac{w_{\eta}}{2} \frac{1}{\tan (\alpha / 2)}
$$

Moreover:

$$
\tan (\alpha) \delta x=\tan (\alpha-\varepsilon)\left(\delta x+w_{\eta}\right)
$$

from which we obtain:

$$
w_{\eta}=\frac{\tan (\alpha) \delta x-\tan (\alpha-\varepsilon) \delta x}{\tan (\alpha-\varepsilon)}
$$

Substituting $w_{\eta}$ in (2), and $h_{\eta}$ in (1) we have:

$$
\tan (3 / 2 \alpha)>\tan (\alpha)+\frac{\tan (\alpha)-\tan (\alpha-\varepsilon)}{2 \tan (\alpha-\varepsilon) \tan (\alpha / 2)}
$$

and with some rearrangements we get:

$$
\tan (\alpha-\varepsilon)>\frac{\tan (\alpha)}{2 \tan (3 / 2 \alpha) \tan (\alpha / 2)-2 \tan (\alpha) \tan (\alpha / 2)+1}
$$

Now, since the tangent function is strictly increasing in $\left(-\frac{\pi}{2}, \frac{\pi}{2}\right)$, we have:

$$
\varepsilon<\hat{\varepsilon}=\alpha-\arctan \left(\frac{\tan (\alpha)}{2 \tan (3 / 2 \alpha) \tan (\alpha / 2)-2 \tan (\alpha) \tan (\alpha / 2)+1}\right)
$$

Since the value of $\varepsilon$ has been chosen strictly smaller than $\hat{\varepsilon}$ the inequality holds. Hence, $\beta_{\mu}<3 / 2 \alpha=\left(\Delta\left(s_{\mu}\right)+1 / 2\right) \alpha$ (since $\left.\Delta\left(s_{\mu}\right)=1\right)$. This completes the proof that Invariant I3 holds.

Lemma 11 Let $\mu$ be an $R$-node different from $\xi$. Then $G_{\mu}$ admits a straightline drawing $\Gamma_{\mu}$ that respects Invariants I1, I2, and $\mathbf{I}$.

Proof: Recall that, by Property 3. (i) the skeleton $\sigma(\mu)$ is isomorphic to $K_{4}$ and it has one crossing; (ii) two children of $\mu$ are $Q$-nodes whose associated edges cross each other in $G_{\mu}$. Hence, denote by $\eta_{1}, \eta_{2}, \eta_{3}$ the three children of $\mu$ whose associated edges of $\sigma(\mu)$ lie on the boundary of the outer face of $\sigma(\mu)$ with $s_{\mu}=s_{\eta_{1}}, t_{\eta_{1}}=s_{\eta_{2}}, t_{\eta_{2}}=s_{\eta_{3}}$, and $t_{\eta_{3}}=t_{\mu}$. Also, denote by $\eta_{4}$ and $\eta_{5}$ the two children of $\mu$ that are $Q$-nodes whose associated edges cross each other in $G_{\mu}$, and so that the poles of $\eta_{4}$ coincide with $t_{\eta_{1}}$ and $t_{\eta_{3}}$, while the poles of $\eta_{5}$ coincide with $t_{\eta_{2}}$ and $s_{\eta_{1}}$. See Figure 25(a) for an illustration. We rotate $\Gamma_{\eta_{1}}$ in such a way that the segment $\overline{s_{\eta_{1}} t_{\eta_{1}}}$ uses the blue slope $b_{2}$. Similarly, we rotate $\Gamma_{\eta_{3}}$ in such a way that the segment $\overline{s_{\eta_{3}} t_{\eta_{3}}}$ uses the blue slope $b_{2 \Delta}$. Furthermore, we scale one of the two drawings so that $t_{\eta_{1}}$ and $s_{\eta_{3}}$ are horizontally aligned. Moreover, we redraw the edge associated with $\eta_{4}$ by using the red slope $r_{2 \Delta}^{+}$ and we redraw the edge associated with $\eta_{5}$ by using the red slope $r_{2}^{-}$. Observe 


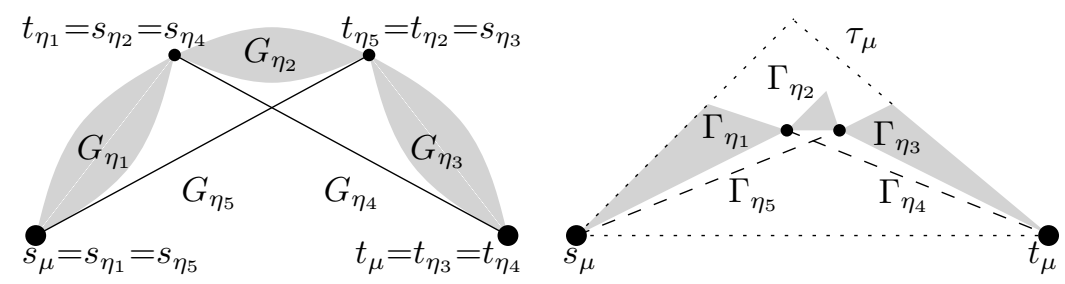

(a)

(b)

Figure 25: Illustration of Lemma 11 . (a) The pertinent graph $G_{\mu}$ of an $R$-node $\mu$. (b) The outer 1-planar drawing of $G_{\mu}$.

that, attaching $\Gamma_{\eta_{4}}$ and $\Gamma_{\eta_{5}}$ to $\Gamma_{\eta_{1}}$ and $\Gamma_{\eta_{3}}$, the length of the segment $\overline{t_{\eta_{1}} s_{\eta_{3}}}$ is determined, because $s_{\eta_{1}}=s_{\eta_{5}}$ and $t_{\eta_{3}}=t_{\eta_{4}}$. Thus, we attach $\Gamma_{\eta_{2}}$ so that $s_{\eta_{2}}$ coincides with $t_{\eta_{1}}$ and that $t_{\eta_{2}}$ coincides with $s_{\eta_{3}}$. See Figure 25(b) for an illustration.

Invariant $\mathbf{I} \mathbf{1}$ and $\mathbf{I} \mathbf{2}$ are respected by construction. Concerning Invariant I3, again by construction $\Gamma_{\mu}$ is contained in a triangle $\tau_{\mu}$ such that $s_{\mu}$ and $t_{\mu}$ are placed at the corners of its base. Moreover, $\beta_{\mu}=\beta_{\eta_{1}}+\alpha$ and $\gamma_{\mu}=\gamma_{\eta_{3}}+\alpha$. Since $\Delta\left(s_{\mu}\right)=\Delta\left(\eta_{1}\right)+1$ and $\Delta\left(t_{\mu}\right)=\Delta\left(\eta_{3}\right)+1$, Invariant $\mathbf{I 3}$ holds.

In the next lemma we process the root of $T$ and its child. Since after that there are no other nodes to be processed, Invariant I3 can be ignored.

Lemma 12 Let $\rho$ be the root of $T$ and let $\xi$ be its unique child. Then $G=$ $G_{\rho} \cup G_{\xi}$ admits a straight-line drawing $\Gamma$ that respects Invariants $\mathbf{I} \mathbf{1}$ and I2.

Proof: Denote by $(s, t)$ the edge associated with $\rho$. Observe that $s=s_{\xi}$ and $t=t_{\xi}$. Recall that $(s, t)$ is an edge of the outer face that is not crossed, thus $\xi$ is such that its reference edge belongs to the outer face of $G_{\xi}^{+}$. If $\xi$ is an $R$-node, then Property 3 holds and we can compute a drawing of $G_{\xi}$ as described in the proof of Lemma 11. Since the horizontal blue slope is not used in $\Gamma_{\xi}$, we can safely add $(s, t)$ by using such a slope, hence, Invariants I1 and I2 hold. If $\xi$ is an $S$-node, then Property 6 holds and we can compute a drawing of $G_{\xi}$ as described in the proof Lemma 8 In order to attach $G_{\rho}$ to $G_{\xi}$, one can observe that the same situation as in case $(i)$ of Property 4 applies, since we need to combine the drawings of a $Q$-node and an $S$-node. We apply for this case the same technique as described in the proof of Lemma 9, for which Invariants I1 and $\mathbf{I} 2$ hold. If $\xi$ is an $S^{*}$-node, then again we can draw $G_{\xi}$ as described in the proof or Lemma 10. Since the horizontal blue slope is not used in $\Gamma_{\xi}$, we can safely add the edge $(s, t)$ by using such a slope. Also in this case Invariants I1 and $\mathbf{I} 2$ hold. If $\xi$ is a $P$-node, then by Property 7 only case (ii) of Property 4 applies. In order to attach $G_{\rho}$ to $G_{\xi}$, one can observe that the same situation as in cases either $(i)$ or $(i i i)$ of Property 4 applies. Hence, we can use the technique described in the proof of Lemma 9 for the specific case, and Invariants I1 and I2 hold. 
Lemma 13 Let $G$ be a biconnected outer 1-planar graph with $n$ vertices and with maximum degree $\Delta$. Then $G$ admits an outer 1-planar straight-line drawing with at most $6 \Delta$ slopes. Also, this drawing can be computed in $O(n)$ time in the real RAM model of computation.

Proof: By Lemmas 8, 9, 10, 11, and 12, $G$ has an outer 1-planar straight-line drawing that maintains the embedding, with at most $6 \Delta$ slopes. Concerning the time complexity, we recall that an outer 1-plane embedding of $G$ can be computed in $O(n)$ time by applying for example one of the algorithms defined in [2, 14. Furthermore, the $S P Q R$-tree of $G$ can be computed in $O(n)$ time [7, and the $S^{*}$-nodes can be created (if any) in $O(n)$ time, as $T$ has $O(n)$ nodes.

Similarly as in the proof of Lemma 6, in order to achieve linear time complexity, we implement the drawing computation phase of BO1P-DrAWER so that it works in two phases. In the first phase we perform a bottom-up visit of $T$ and for each node $\mu$, we compute a drawing of the frame of $\mu$ for $S$-nodes and $R$-nodes or we combine the drawings of the frames of the children of $\mu$ for $P$-nodes. At the end of this phase, each node of $T$ is associated with a drawing of its frame (for $S$-nodes and $R$-nodes) or with a drawing of the union of the frames of its children (for $P$-nodes and $S^{*}$-nodes). The drawing associated with each node $\mu$ can be computed in $O\left(n_{\mu}^{*}\right)$, where $n_{\mu}^{*}$ is the number of vertices in the frame of $\mu$. The total number of vertices and edges of all the skeletons of $T$ is $O(n)$ [7. Thus, $\sum_{\mu \in T} O\left(n_{\mu}^{*}\right)=O(n)$ and the first phase can be executed in $O(n)$ time. In the second phase we perform a top-down visit of $T$ and compute the final coordinates of each vertex of $G$, thus combining all the drawings computed in the first phase. Once the coordinates of the vertices of the drawing associated with $\mu$ have been fixed, we can fix the coordinates of the vertices of the drawings associated with its children (or its grand-children in the case of $P$ nodes and $S^{*}$-nodes). Again, since $\sum_{\forall \mu \in T} O\left(n_{\mu}^{*}\right)=O(n)$, the time complexity of this phase is $O(n)$. The statement about the time complexity follows.

Extension to Connected Graphs. In order to deal with simply connected graphs we use the technique described in Section 3 that augments a simply connected outer 1-planar graph with maximum degree $\Delta$ to a biconnected outer 1-planar graph with maximum degree $\Delta+2$. By applying BO1P-DrAWER to the resulting biconnected graph and removing the inserted edges we obtain the following result.

Theorem 2 Let $G$ be an outer 1-planar graph with $n$ vertices and with maximum degree $\Delta$. Then $G$ admits an outer 1-planar straight-line drawing with at most $6 \Delta+12$ slopes. Also, this drawing can be computed in $O(n)$ time in the real RAM model of computation.

\section{Conclusions and Open Problems}

In this paper we proved a quadratic upper bound on the planar slope number of outer 1-planar graphs. This result improves, for this family, the $O\left(n^{5}\right)$ upper 
bound that holds for general planar partial 3-trees. We also initiated the study of the slope number of "nearly-planar" graphs. In particular, we proved a linear upper bound on the outer 1-planar slope number of outer 1-planar graphs. This result generalizes the result on the outerplanar slope number of outerplanar graphs. Several interesting problems are suggested by the results in this paper.

- There is still a gap between upper and lower bound on the planar slope number of outer 1-planar graphs, which can be closed in either direction.

- An obvious extension of our study of the outer 1-planar slope number is the investigation of the 1-planar slope number. Is the 1-planar slope number of 1-planar straight-line drawable graphs (not all 1-planar graphs admit a 1-planar straight-line drawing [15]) bounded in $\Delta$ ?

- Finally, it could be interesting to further explore trade-offs between slopes and crossings, e.g., can we draw planar partial 3-trees with $o\left(\Delta^{5}\right)$ slopes and a constant number of crossings per edge?

\section{Acknowledgments}

We thank the anonymous reviewers for their valuable comments, which helped us to improve the quality of the paper. 


\section{References}

[1] M. J. Alam, F. J. Brandenburg, and S. G. Kobourov. Straight-line grid drawings of 3-connected 1-planar graphs. In S. K. Wismath and A. Wolff, editors, GD 2013, volume 8242 of LNCS, pages 83-94. Springer, 2013. doi:10.1007/978-3-319-03841-4_8.

[2] C. Auer, C. Bachmaier, F. J. Brandenburg, A. Gleißner, K. Hanauer, D. Neuwirth, and J. Reislhuber. Recognizing outer 1-planar graphs in linear time. In S. Wismath and A. Wolff, editors, GD 2013, volume 8242 of $L N C S$, pages 107-118. Springer, 2013. doi:10.1007/978-3-319-03841-4_10.

[3] M. J. Bannister, S. Cabello, and D. Eppstein. Parameterized complexity of 1-planarity. In F. Dehne, R. Solis-Oba, and J.-R. Sack, editors, WADS 2013, volume 8037 of $L N C S$, pages 97-108. Springer, 2013. doi:10.1007/ 978-3-642-40104-6_9.

[4] J. Barát, J. Matousek, and D. R. Wood. Bounded-degree graphs have arbitrarily large geometric thickness. The Electronic Journal of Combinatorics, 13(1), 2006.

[5] F.-J. Brandenburg, D. Eppstein, A. Gleißner, M. T. Goodrich, K. Hanauer, and J. Reislhuber. On the density of maximal 1-planar graphs. In W. Didimo and M. Patrignani, editors, GD 2012, volume 7704 of LNCS, pages 327-338. Springer, 2012. doi:10.1007/978-3-642-36763-2_29.

[6] H. R. Dehkordi and P. Eades. Every outer-1-plane graph has a right angle crossing drawing. International Journal on Computational Geometry and Applications, 22(6):543-558, 2012. doi:10.1142/S021819591250015X.

[7] G. Di Battista and R. Tamassia. On-line planarity testing. SIAM Journal on Computing, 25(5):956-997, 1996. doi:10.1137/S0097539794280736.

[8] E. Di Giacomo, W. Didimo, G. Liotta, H. Meijer, F. Trotta, and S. K. Wismath. k-colored point-set embeddability of outerplanar graphs. Journal of Graph Algorithms and Applications, 12(1):29-49, 2008. doi:10.7155/ jgaa.00158.

[9] E. Di Giacomo, G. Liotta, and F. Montecchiani. The planar slope number of subcubic graphs. In A. Pardo and A. Viola, editors, LATIN 2014, volume 8392 of $L N C S$, pages 132-143. Springer, 2014. doi:10.1007/ 978-3-642-54423-1_12.

[10] W. Didimo. Density of straight-line 1-planar graph drawings. Information Processing Letters, 113(7):236-240, 2013. doi:10.1016/j.ipl.2013.01. 013.

[11] V. Dujmović, M. Suderman, and D. R. Wood. Graph drawings with few slopes. Comput. Geom., 38(3):181-193, 2007. doi:10.1016/j.comgeo. 2006.08 .002 
740 Di Giacomo et al. Drawing Outer 1-planar Graphs with Few Slopes

[12] P. Eades, S.-H. Hong, N. Katoh, G. Liotta, P. Schweitzer, and Y. Suzuki. A linear time algorithm for testing maximal 1-planarity of graphs with a rotation system. Theoretical Computer Science, 513(0):65 - 76, 2013. doi:10.1016/j.tcs.2013.09.029.

[13] P. Eades and G. Liotta. Right angle crossing graphs and 1-planarity. Discrete Applied Mathematics, 161(78):961 - 969, 2013. doi:10.1016/j.dam. 2012.11.019.

[14] S.-H. Hong, P. Eades, N. Katoh, G. Liotta, P. Schweitzer, and Y. Suzuki. A linear-time algorithm for testing outer-1-planarity. Algorithmica, pages 1-22, 2014. doi:10.1007/s00453-014-9890-8

[15] S.-H. Hong, P. Eades, G. Liotta, and S.-H. Poon. Fáry's theorem for 1planar graphs. In J. Gudmundsson, J. Mestre, and T. Viglas, editors, COCOON 2012, volume 7434 of LNCS, pages 335-346. Springer, 2012. doi:10.1007/978-3-642-32241-9_29.

[16] V. Jelínek, E. Jelínková, J. Kratochvíl, B. Lidický, M. Tesar, and T. Vyskocil. The planar slope number of planar partial 3-trees of bounded degree. Graphs and Combinatorics, 29(4):981-1005, 2013. doi:10.1007/ s00373-012-1157-z.

[17] B. Keszegh, J. Pach, and D. Pálvölgyi. Drawing planar graphs of bounded degree with few slopes. SIAM Journal on Discrete Mathematics, 27(2):1171-1183, 2013. doi:10.1137/100815001.

[18] K. B. Knauer, P. Micek, and B. Walczak. Outerplanar graph drawings with few slopes. Computational Geometry, 47(5):614-624, 2014. doi:10.1016/ j.comgeo.2014.01.003.

[19] V. P. Korzhik and B. Mohar. Minimal obstructions for 1-immersions and hardness of 1-planarity testing. Journal of Graph Theory, 72(1):30-71, 2013. doi:10.1002/jgt.21630.

[20] W. Lenhart, G. Liotta, D. Mondal, and R. Nishat. Planar and plane slope number of partial 2-trees. In S. Wismath and A. Wolff, editors, GD 2013, volume 8242 of $L N C S$, pages 412-423. Springer, 2013. doi: 10.1007/978-3-319-03841-4_36.

[21] P. Mukkamala and D. Pálvölgyi. Drawing cubic graphs with the four basic slopes. In M. Kreveld and B. Speckmann, editors, GD 2011, volume 7034 of $L N C S$, pages 254-265. Springer, 2012. doi:10.1007/ 978-3-642-25878-7_25.

[22] J. Pach and D. Pálvölgyi. Bounded-degree graphs can have arbitrarily large slope numbers. The Electronic Journal of Combinatorics, 13(1), 2006.

[23] J. Pach and G. Tóth. Graphs drawn with few crossings per edge. Combinatorica, 17(3):427-439, 1997. doi:10.1007/BF01215922 
[24] F. P. Preparata and M. I. Shamos. Computational Geometry: An Introduction. Springer-Verlag New York, Inc., New York, NY, USA, 1985.

[25] Y. Suzuki. Re-embeddings of maximum 1-planar graphs. SIAM Journal on Discrete Mathematics, 24(4):1527-1540, 2010. doi:10.1137/090746835

[26] G. A. Wade and J.-H. Chu. Drawability of complete graphs using a minimal slope set. The Computer Journal, 37(2):139-142, 1994. doi:10.1093/ comjnl/37.2.139. 